\title{
Article
}

\section{Top-Bottom Condensation Model: Symmetries and Spectrum of the Induced 2HDM}

\author{
Alexander A. Osipov ${ }^{1}\left[\right.$, Brigitte Hiller ${ }^{2, *}$, Alex H. Blin ${ }^{2} \mathbb{D}$ and Marcos Sampaio ${ }^{3}$ \\ 1 Bogoliubov Laboratory of Theoretical Physics, JINR, 141980 Dubna, Russia; aaosipov@jinr.ru \\ 2 CFisUC, Department of Physics, University of Coimbra, P-3004-516 Coimbra, Portugal; alex@uc.pt \\ 3 CCNH Centro de Ciências Naturais e Humanas, Universidade Federal do ABC, \\ Santo André 09210-580, SP, Brazil; marcos.sampaio@ufabc.edu.br \\ * Correspondence: brigitte@fis.uc.pt
}

Citation: Osipov, A.A.; Hiller, B.; Blin, A.H.; Sampaio, M. Top-Bottom Condensation Model: Symmetries and Spectrum of the Induced 2HDM. Symmetry 2021, 13, 1130. https:// doi.org/10.3390/sym13071130

Academic Editors: Zoltán Trócsányi, Adam Kardos and Giuseppe Bevilacqua

Received: 21 May 2021

Accepted: 18 June 2021

Published: 24 June 2021

Publisher's Note: MDPI stays neutral with regard to jurisdictional claims in published maps and institutional affiliations.

Copyright: (C) 2021 by the authors. Licensee MDPI, Basel, Switzerland. This article is an open access article distributed under the terms and conditions of the Creative Commons Attribution (CC BY) license (https:/ / creativecommons.org/licenses/by/ $4.0 /)$.

\begin{abstract}
Here, we use the Schwinger-DeWitt approach to address the four-fermion composite Higgs effective model proposed by Miransky, Tanabashi and Yamawaki (MTY). The surprising benefit of such an approach is that it is possible to ascribe to a SM-type Higgs a quark-antiquark structure of predominantly a $\bar{b} b$ nature with a small $\bar{t} t$ admixture, which in turn yields a Higgs mass compatible with the observed value of $125 \mathrm{GeV}$. We discuss this result in a detailed and pedagogical way, as it goes against the common belief that this model and akin composite descriptions should predict a Higgs mass-of-order of twice the top quark mass, contrary to empirical evidence. A further aspect of this approach is that it highlights the link of the $S U(2)_{L} \times U(1)_{R}$ symmetric four-fermion MTY model interactions of the heavy quark family to a specific two-Higgs-doublet model (2HDM), and the necessity to go beyond the one Higgs doublet to obtain the empirical Higgs mass within composite models. By appropriately fixing the symmetry-defining interaction parameters, we show that the resulting CP-preserving spectrum harbors the following collective states at the electroweak scale $\Lambda_{E W}=246 \mathrm{GeV}$ : a light scalar to which the standard Higgs is associated; a heavier neutral state preconized as the Nambu partner of the standard Higgs within the Nambu sum rule; the expected triplet of Goldstone bosons associated with the longitudinal polarizations of the electroweak massive bosons; and a neutral pseudoscalar state that in the limit of a global $U(1)_{A}$ symmetry would be a Goldstone mode. The anomalous breaking of this axial symmetry is a subleading effect in a large $N_{c}$ counting scheme, and we discuss how it modifies the leading-order Nambu sum rule result and its relevance for the qualitative description of the spectrum.
\end{abstract}

Keywords: composite higgs model; collective state spectrum; dynamical symmetry breaking; effective action

\section{Introduction}

Symmetry principles based on space-time and internal (local) symmetries of the fields play a central role in particle physics.The Standard Model (SM) is a theory based on the gauge groups $S U(3)_{c} \otimes S U(2)_{L} \otimes U(1)_{Y}$, which is realised in three phases: the Coulomb phase (photons), the Higgs phase (W, Z) and the confinement phase (gluons). Whereas the electroweak mediators appear explicitly in the spectrum, the gluons may only appear as bound states.

Global symmetries are usually approximate, or can be broken (either at Lagrangian level or via an anomaly).

Anomalously broken gauge symmetries are usually restored by anomaly cancellation mechanisms in the Standard Model. However, a gauge symmetry can be spontaneously broken through the Higgs-Brout-Englert mechanism. The $S U(2)_{L} \otimes U(1)_{Y}$ symmetry is still present, but since the vacuum is not symmetric, it is not apparent. The spontaneous breaking of the electroweak symmetry generates the masses of the vector bosons $W^{ \pm}, Z^{0}$ in a gauge-invariant way, while the photon remains massless and thus still explicitly preserves 
$U(1)$ gauge invariance. The Higgs mechanism assumes the existence of an isospin doublet of complex scalar fields,

$$
\Phi=\left(\begin{array}{c}
\Re \Phi^{+}+i \Im \Phi^{+} \\
\Re \Phi^{0}+i \Im \Phi^{0}
\end{array}\right)
$$

associated with the potential $V(\Phi)=-\mu^{2} \Phi^{\dagger} \Phi+\lambda\left(\Phi^{\dagger} \Phi\right)^{2}$ invariant under $S U(2)_{L} \otimes U(1)_{Y}$ and $\mu^{2}, \lambda>0$. The Mexican hat-shaped potential has a minimum at $\langle\Phi\rangle \equiv v=\sqrt{-\mu^{2} / \lambda}$, which is fixed by experimental parameters at $v \approx 246 \mathrm{GeV}$. Thus, $S U(2)_{L} \otimes U(1)_{Y}$ is hidden, as the vacuum is not symmetric. For each fermion field of definite right or left chirality, the interaction with the scalar field $\Phi$ generates their masses after spontaneous symmetrybreaking, excluding the neutrinos which are only left-handed. From the four initial degrees of freedom of the field $\Phi$, three are used $W^{ \pm}, Z^{0}$ gauge bosons to acquire their masses and the other one corresponds to a physical particle, the Higgs boson $H$. The Higgs boson stands out from the other particles in some ways. It is a spin 0 matter boson that does not mediate gauge interactions, and its coupling strength to matter particles defines how massive they are. Theoretical constraints, as well as direct searches and precision measurements (for recent reviews, see [1,2]) on the Higgs boson announced by CERN in 2012 are compatible with the mass value $125.10 \pm 0.14 \mathrm{GeV}$ [3].

The SM is an effective QFT which is perturbative up to the Planck scale where quantum gravity effects kick in. Thus, we expect a cutoff scale of the SM to be (at most) at the Planck mass. Because the dimensionful parameter $\mu^{2}$ is unnaturally small (even if it is tuned small, at tree level, loop contributions are quadratically divergent), we conclude that the SM is very fine-tuned. In mathematical terms, this is put as follows. The Higgs mass term is an operator of dimension $d=2$, which means that it has a coefficient of dimension $1 / \Lambda_{S M}^{d-4}$, and thus, $\mu^{2}=k \Lambda_{S M}^{2} \Phi^{\dagger} \Phi$ where $k$ is a numerical coefficient. Taking $\mu^{2}=M_{H}^{2} / 2 \approx(88 \mathrm{GeV})^{2}$ and $\Lambda_{S M}=M_{G U T} \approx 10^{15} \mathrm{GeV}$, then $k \approx 10^{-26} \lll 1$. This is the hierarchy problem.

There are basically two ways to avoid the hierarchy problem of the SM: weakly and strongly coupled theories. One avenue assumes an elementary Higgs and new symmetries, and the other one assumes new interactions and that the Higgs is a composite particle. Supersymmetry is an example of weakly coupled theories: the quadratic divergence due to the quadratic quantum correction is cancelled by the contribution from a superpartner particle. On the other hand, a typical example of strongly coupled models is Composite Higgs Models (CHMs).

Once we assume a composite Higgs model, we ought to work out the strong dynamics that are responsible for the production of such a composite state and evaluate its predictions. For instance, in a theory with strong dynamics, one has a mass spectrum with a dense tower of resonances built from the more fundamental objects that interact strongly. Two scenarios are usually contemplated: one in which the Higgs is a light scalar (dilaton-like) particle of the new strong dynamics, or it arises similar to pions in QCD as a pseudoNambu-Goldstone boson (PNGB) [4,5]. In this case, strong dynamics will generate, among other bound states, a PNGB with the quantum numbers of the Higgs, which breaks the electroweak symmetry.

Thus, we use symmetry to explain the mass of the composite Higgs boson because, being a PNGB, this Higgs particle will be light. Its mass will not get radiative corrections above the compositeness scale. In the 1980s, Georgi and Kaplan [6-8] pointed out that a Higgs boson resulting from a bound state due to strong dynamics at some energy scale $f$ larger than the electroweak (TeV) scale would be less massive as compared to other resonances. One can think of $f$ in analogy to the weak decay constant of the pion $f_{\pi}$.

The scale of such new strong dynamics is typically much higher than the energies that can be achieved at current experiments. Thus, one studies the low-energy phenomenology of these models in a way that does not depend on the specific high-energy physics through an effective Lagrangian approach. To describe an underlying theory that is only probed at energy scales $E<\Lambda$, a particle of mass $m>\Lambda$ cannot be produced as a real state. It 
can only contribute through short-distance virtual effects. The generating functional for Green's functions leads to an effective theory after "integrating out" the quantum fluctuations due to the heavier particles, after which only the degrees of freedom relevant to the energy scale are considered. A typical example of models that are strongly interacting and describes a composite Higgs boson is Technicolor (TC) [9]. TC assumes a new SU(N) TC gauge symmetry describing the dynamics of new stronginteractions in analogy to the usual $S U(3)_{C}$ gauge interactions of QCD. Additionally, TC Lagrangian is assumed to have the electroweak chiral symmetry that is broken spontaneously to the custodial symmetry group by the techniquark condensate $\left\langle 0\left|\bar{q}_{T C} q_{T C}\right| 0\right\rangle \neq 0 \Rightarrow S U(2)_{L} \otimes S U(2)_{R} \rightarrow S U(2)_{L+R}$, resulting in three Goldstones bosons (technipions). Upon gauging the electroweak subgroup $S U(2)_{L} \otimes U(1)_{Y}$, such technipions disappear from the spectrum giving place to longitudinal bosons $W_{L}^{ \pm}, Z_{L}$ which get their mass through the Higgs mechanism without the appearance of an elementary Higgs. Although TC is appealing, it turns out that, at least in the simpler setups, they are very constrained by measurements of electroweak precision observables and the heavy quark masses, in addition to the production of Flavor Changing Neutral Currents (FCNC).

A large variety of models based on an extended (non-minimal) Higgs sector have been proposed. The mechanism of electroweak symmetry breaking with one doublet is not confirmed, and one may consider larger representations motivated by higher-scale symmetries or phenomenological arguments, such as new sources of CP violation [10]. Two-Higgs-doublet models (2HDM) require the introduction of a second Higgs doublet. The Minimal Supersymmetric Standard Model (MSSM) requires a second Higgs doublet to warrant cancellation of gauge anomalies. The Higgs sector of the MSSM is described by a 2HDM containing two chiral Higgs supermultiplets that differ by the sign of hypercharge. As for CP symmetry, it can be violated explicitly in the scalar sector if some of the coefficients in the 2HDM potential are complex and survive by neutral-flavour conservation requirements. Moreover, a specific 2HDM called the inert model [11] admits a 2HD extension of the SM scalar sector that could be a candidate to explain dark matter.

Finally, the fermion mass spectrum in the SM is related to the Yukawa couplings between the Higgs field and the fermions. Such couplings are arbitrary, and thus cannot explain why there are three generations of fermions and their large spectrum of masses. In a model with one doublet, all quarks receive their masses from the same doublet. In a 2HDM, the Yukawa coupling is more natural. For instance, it is possible to generate Yukawa couplings such that the bottom quark $(\approx 4 \mathrm{GeV})$ receives its mass from one doublet, while the top quark $(\approx 170 \mathrm{GeV})$ gets it from another doublet.

In the present work, we concentrate on the top-condensation description of compositeness, which dates back to the works [12-14] and has been steadily developed by [15-25] ever since. In this approach, the elementary SM Higgs is replaced by a bound state with an underlying top-antitop structure. It is assumed that below a large-scale $\Lambda$, eventually of the grand unification (GUT), the effective Lagrangian is described by four quark interactions subject to critical behavior that lead to condensation and emergence of mass beyond the SM. However, the SM Higgs mass $m_{\chi}$ within such an approach persists systematically in being larger than the empirical value, $\sqrt{2} m_{t}<m_{\chi}<2 m_{t}$, with $m_{t}=173 \mathrm{GeV}$ denoting the mass of the $t$ quark. The upper boundary is obtained in the large $N_{c}$ approximation for one Higgs doublet [19], and also in the 2HDM studies of [20,21]. The lower emerges upon using renormalization group equations that take gauge and Higgs fields into account at one-loop order $[19,20]$.

Another avenue to deal with the too-high Higgs mass uses the top-seesaw mechanism, refs. [26-28].

The relation $m_{\chi}=2 m_{t}$ is a well-known result within the Nambu-Jona-Lasinio (NJL) model $[29,30]$ that predicts the mass of the scalar bound state to be twice the quark mass when chiral symmetry is spontaneously broken. This relation is generalized to $m_{1}^{2}+m_{2}^{2}=4 m_{q}^{2}$ within the Nambu sum rule [31-33], when two collective states (called Nambu partners), each of masses $m_{1}$ and $m_{2}$, are present in the spectrum. A consequence of 
the sum rule is that at least two Higgs doublets are required to obtain the phenomenological mass value of the light Higgs. In this scenario, a Nambu partner for the light Higgs is obtained with mass $325 \mathrm{GeV}$ [33].

Although it is general consensus (see, e.g., refs. [34,35]) that standard 2HDM for topcondensation $[17,20,21]$ are unable to yield the SM Higgs mass, we show here that in the model [17], the mass $m_{\chi}$ is obtained in the interval $2 m_{b}<m_{\chi}<m_{t}$.

The paper is organized as follows. In Section 2 we present the most important features of the model [17] and address its symmetry content in different stages that prepare for the new aspect of our approach, which is the derivation of the low-energy effective action of the model on the basis of the Schwinger-DeWitt background field method, presented in Section 3. This includes the introduction of relevant bosonic variables with a definite quark content, in terms of which two Higgs doublets are characterized. In Section 4, we derive through the asymptotic proper time expansion the Higgs sector related gap equations, quark condensates and masses, and discuss the phenomenon of bottom quark mass catalysis. The induced interaction potential involving cubic and quartic interactions among the members of the two Higgs doublets is derived in this section and displayed in the Higgs basis, as well as the Yukawa couplings. The spectrum and role of coupling constants of the theory are addressed in Section 5, together with the Nambu sum rule. It is shown that the exact form of this sum rule is obtained in the model at leading order in a large $N_{C}$ expansion, but that the $U(1)_{A}$ anomaly breaks the pattern, since it is a subleading effect in $N_{c}$ counting, causing a modification. Numerical estimates for the spectrum are obtained and discussed. After considering the effects induced in the gauge sector, Section 6, we discuss numerical estimates of our results in Section 7 and conclude in Section 8. In Appendix A are relevant details of diagonalization.

\section{Lagrangian and Symmetries}

We discuss in this section the general features of the four-quark interaction model [17] using for simplicity only the top and bottom quarks. Our guiding line will be to make sure that the symmetry content is kept intact in all steps that lead to the low energy effective action obtained by the Schwinger-DeWitt expansion. This first involves the introduction of a set of auxiliary bosonic variables in the fermionic fuctional integral in an invariant way, which linearizes the interactions and simplifies the task of integrating out the short distance components of the fermionic degrees of freedom within the background field method of Schwinger-DeWitt. We show how these bosonic variables relate to two Higgs doublets with a definite quark content, on the basis of the symmetry transformation properties. These results will be used in the following sections to obtain the induced scale-dependent kinetic terms and quartic interactions for the bosons and to cast the low-energy effective Lagrangian in the standard form involving two Higgs doublets, from which the analysis of dynamical symmetry breaking and spectrum will follow.

\subsection{Effective Fermion-Gauge-Boson Lagrangian}

Although we will focus on the fermionic interactions that give rise to the composite Higgs sector, we indicate for completeness the full Lagrangian that has been considered within the present approach, and refer for more details to [36]. We start with the gauged fermionic system with $S U(2)_{L} \times U(1)_{R}$ local symmetry

$$
\mathcal{L}=\bar{\psi}_{L} i \gamma^{\mu} \mathcal{D}_{\mu} \psi_{L}+\sum_{a=1}^{2} \bar{\psi}_{R}^{a} i \gamma^{\mu} \mathcal{D}_{\mu} \psi_{R}^{a}+\mathcal{L}_{\mathrm{YM}}+\mathcal{L}_{4 \psi},
$$

involving only the top and bottom quarks for simplicity

$$
\psi=\left(\begin{array}{l}
\psi_{1} \\
\psi_{2}
\end{array}\right)=\left(\begin{array}{l}
t \\
b
\end{array}\right)
$$


Their chiral projections $\psi_{L, R}=P_{L, R} \psi$ are defined through the operators $P_{R}=\frac{1}{2}\left(1+\gamma_{5}\right)$, $P_{L}=\frac{1}{2}\left(1-\gamma_{5}\right)$. The covariant derivatives are given by the SM expressions

$$
\begin{aligned}
& \mathcal{D}_{\mu} \psi_{L}=\left(\partial_{\mu}-i g_{R} T_{i} A_{R \mu}^{i}-i g_{R}^{\prime} Y_{L} B_{R \mu}\right) \psi_{L}, \\
& \mathcal{D}_{\mu} \psi_{R}=\left(\partial_{\mu}-i g_{R}^{\prime} Q B_{R \mu}\right) \psi_{R},
\end{aligned}
$$

where $A_{R \mu}=A_{R \mu}^{i} T_{i}$ and $B_{R \mu}$ are respectively the gauge fields associated to the $S U(2)_{L}$ and $U(1)_{R}$ groups. The electric charges of the quarks are given in units of $e>0$ through the matrix $Q=T_{3}+Y_{L}$ in terms of the hypercharge $Y_{L}=1 / 6$ and the third component of the Lie algebra generators $T_{i}=\tau_{i} / 2, i=1,2,3$, with $\tau_{i}$ the Pauli matrices.

The Yang-Mills part $\mathcal{L}_{\mathrm{YM}}$ is standard

$$
\mathcal{L}_{\mathrm{YM}}=-\frac{1}{4}\left(B_{R \mu v}^{2}+\vec{G}_{R \mu v}^{2}\right),
$$

where

$$
\begin{aligned}
B_{R \mu v} & =\partial_{\mu} B_{R v}-\partial_{\nu} B_{R \mu}, \\
\vec{G}_{R \mu v} & =\partial_{\mu} \vec{A}_{R v}-\partial_{\nu} \vec{A}_{R \mu}+g_{R} \vec{A}_{R \mu} \times \vec{A}_{R v} .
\end{aligned}
$$

As will be discussed in Section 6, the gauge fields are still subject to a rescaling $\vec{A}_{R \mu}=Z_{A}^{-1 / 2} \vec{A}_{\mu}, B_{R \mu}=Z_{B}^{-1 / 2} B_{\mu}$, leading to the physical fields $\vec{A}_{\mu}, B_{\mu}$.

The effective four-fermion interaction $\mathcal{L}_{4 \psi}$ is assumed to arise at a high energy scale $\Lambda$ and will give origin to the composite Higgs system. It has the most general $S U(2)_{L} \times U(1)_{R}$ group structure [17]

$$
\begin{aligned}
\mathcal{L}_{4 \psi} & =g_{1}\left(\bar{\psi}_{L}^{a} \psi_{R}^{b}\right)\left(\bar{\psi}_{R}^{b} \psi_{L}^{a}\right) \\
& +g_{2}\left(\bar{\psi}_{L}^{a} \psi_{R}^{b}\right)\left(i \tau_{2}\right)^{a c}\left(i \tau_{2}\right)^{b e}\left(\bar{\psi}_{L}^{c} \psi_{R}^{e}\right) \\
& +g_{3}\left(\bar{\psi}_{L}^{a} \psi_{R}^{b}\right) \tau_{3}^{b c}\left(\bar{\psi}_{R}^{c} \psi_{L}^{a}\right)+\text { h.c. }
\end{aligned}
$$

with implicit summation over the color degrees of freedom (not explicitly shown) taken for each pair of quarks within parentheses. The couplings $g_{i}$ are considered to be real and positive, with dimension $\left[g_{i}\right]=M^{-2}$, and the symmetry content associated to the corresponding interactions is

$$
\begin{array}{ll}
g_{1}: & S U(3)_{c} \times S U(2)_{L} \times S U(2)_{R} \times U(1)_{V} \times U(1)_{A} . \\
g_{2}: & S U(3)_{c} \times S U(2)_{L} \times S U(2)_{R} \times U(1)_{V} . \\
g_{3}: & S U(3)_{c} \times S U(2)_{L} \times U(1)_{R} \times U(1)_{V} \times U(1)_{A} .
\end{array}
$$

This can perhaps be better seen by introducing, in the following, the variables $s_{\alpha}, p_{\alpha}$ (the Greek index $\alpha$ takes the values $\alpha=0,1,2,3$, and $\tau_{0}=1$ ), which will also be an asset to assert in the next section the functional equivalence of the fermionic Lagrangian density associated with Equation (9) and a semi-bosonized Lagrangian involving Yukawalike interactions,

$$
\begin{aligned}
& \bar{\psi} \tau_{\alpha} \psi=\tau_{\alpha}^{a b}\left(\bar{\psi}_{L}^{a} \psi_{R}^{b}+\bar{\psi}_{R}^{a} \psi_{L}^{b}\right) \equiv s_{\alpha}, \\
& \bar{\psi} i \gamma_{5} \tau_{\alpha} \psi=i \tau_{\alpha}^{a b}\left(\bar{\psi}_{L}^{a} \psi_{R}^{b}-\bar{\psi}_{R}^{a} \psi_{L}^{b}\right) \equiv p_{\alpha},
\end{aligned}
$$

and define the complex $2 \times 2$ matrix 


$$
\begin{aligned}
& \Sigma_{a b}=\frac{1}{2}\left(s_{\alpha}+i p_{\alpha}\right) \tau_{\alpha}^{a b}=2 \bar{\psi}_{R}^{b} \psi_{L}^{a}, \\
& \Sigma_{a b}^{\dagger}=\frac{1}{2}\left(s_{\alpha}-i p_{\alpha}\right) \tau_{\alpha}^{a b}=2 \bar{\psi}_{L}^{b} \psi_{R}^{a} .
\end{aligned}
$$

Then, one can easily see that the term proportional to $g_{1}$ in Equation (9) can be written as

$$
\begin{aligned}
\left(\bar{\psi}_{L}^{a} \psi_{R}^{b}\right)\left(\bar{\psi}_{R}^{b} \psi_{L}^{a}\right) & =\frac{1}{4} \Sigma_{b a}^{\dagger} \Sigma_{a b}=\frac{1}{4} \operatorname{tr}\left(\Sigma^{\dagger} \Sigma\right)=\frac{1}{8}\left(s_{\alpha}^{2}+p_{\alpha}^{2}\right) \\
& =\frac{1}{8}\left[\left(\bar{\psi} \tau_{\alpha} \psi\right)^{2}+\left(\bar{\psi} i \gamma_{5} \tau_{\alpha} \psi\right)^{2}\right] .
\end{aligned}
$$

The NJL-model with chiral $U(2)_{L} \times U(2)_{R}$ symmetry emerges when only the coupling $g_{1}$ is kept, leading in the Nambu-Goldstone phase to a set of four massless pseudoscalars and four mass-degenerate scalars with a mass twice the constituent fermion mass.

The term proportional to $g_{2}$ of Equation (9) yields, after using

$$
\left(i \tau_{2}\right)^{a b}\left(i \tau_{2}\right)^{c d}=\epsilon_{a b} \epsilon_{c d},
$$

the following expression

$$
\begin{aligned}
& \left(\bar{\psi}_{L}^{a} \psi_{R}^{c}\right)\left(i \tau_{2}\right)^{a b}\left(i \tau_{2}\right)^{c d}\left(\bar{\psi}_{L}^{b} \psi_{R}^{d}\right)=\frac{1}{4} \Sigma_{c a}^{+} \Sigma_{d b}^{\dagger} \epsilon_{a b} \epsilon_{c d}=\frac{1}{2} \operatorname{det} \Sigma^{\dagger} \\
& =\frac{1}{8}\left[s_{0}^{2}-s_{i}^{2}-p_{0}^{2}+p_{i}^{2}-2 i\left(s_{0} p_{0}-s_{i} p_{i}\right)\right]
\end{aligned}
$$

leading to

$$
\begin{aligned}
& \left(\bar{\psi}_{L}^{a} \psi_{R}^{c}\right)\left(i \tau_{2}\right)^{a b}\left(i \tau_{2}\right)^{c d}\left(\bar{\psi}_{L}^{b} \psi_{R}^{d}\right)+\text { h.c. } \\
& =\frac{1}{2}\left(\operatorname{det} \Sigma^{\dagger}+\operatorname{det} \Sigma\right)=\frac{1}{4}\left[s_{0}^{2}-s_{i}^{2}-p_{0}^{2}+p_{i}^{2}\right] \\
& =\frac{1}{4}\left[\left(\bar{\psi} \tau_{0} \psi\right)^{2}-\left(\bar{\psi} \tau_{i} \psi\right)^{2}-\left(\bar{\psi} i \gamma_{5} \tau_{0} \psi\right)^{2}+\left(\bar{\psi} i \gamma_{5} \tau_{i} \psi\right)^{2}\right] .
\end{aligned}
$$

One recognizes that the global $U(1)_{A}$ symmetry is absent. Two distinct combinations of fermionic bilinears emerge, each fulfilling $S U(2)_{L} \times S U(2)_{R}$ chiral symmetry, one involving a scalar isospin singlet and pseudo scalar isospin triplet, the other with opposite isotopic assignments and signs. Only this determinantal interaction lifts the $U(1)_{A}$ symmetry in the Lagrangian density Equation (2), without breaking chiral symmetry, as known from the seminal works of ' $t$ Hooft [37]. Its presence is required as a massless Nambu-Goldstone mode that would occur after spontaneous breaking of the $U(1)_{A}$ symmetry has not been observed experimentally. Finally, for the term proportional to $g_{3}$ in Equation (9), one has

$$
\begin{aligned}
\left(\bar{\psi}_{L}^{a} \psi_{R}^{b}\right) \tau_{3}^{b c}\left(\bar{\psi}_{R}^{c} \psi_{L}^{a}\right)=\frac{1}{4} \Sigma_{b a}^{+} \tau_{3}^{b c} \Sigma_{a c}=\frac{1}{4} \operatorname{tr}\left(\Sigma^{\dagger} \Sigma \tau_{3}\right) \\
=\frac{1}{4}\left(s_{0} s_{3}-s_{1} p_{2}+s_{2} p_{1}+p_{0} p_{3}\right) \\
=\frac{1}{4}\left[\left(\bar{\psi} \tau_{0} \psi\right)\left(\bar{\psi} \tau_{3} \psi\right)-\left(\bar{\psi} \tau_{1} \psi\right)\left(\bar{\psi} i \gamma_{5} \tau_{2} \psi\right)\right. \\
\left.\quad+\left(\bar{\psi} \tau_{2} \psi\right)\left(\bar{\psi} i \gamma_{5} \tau_{1} \psi\right)+\left(\bar{\psi} i \gamma_{5} \tau_{0} \psi\right)\left(\bar{\psi} i \gamma_{5} \tau_{3} \psi\right)\right] .
\end{aligned}
$$

This interaction violates isospin and spatial parity, as expected from a $S U(2)_{L} \times U(1)_{R}$ symmetry content. Here, the charge-neutral fermionic bilinears combine only in pairs 
involving the same parity, as opposed to the charged bilinears. Combining Equations (15), (18) and (19) in (9) one obtains

$$
\begin{aligned}
\mathcal{L}_{4 \psi}= & \frac{1}{4}\left[\left(g_{1}+g_{2}\right)\left(p_{0}^{2}+s_{i}^{2}\right)+\left(g_{1}-g_{2}\right)\left(s_{0}^{2}+p_{i}^{2}\right)\right. \\
& \left.+2 g_{3}\left(p_{0} p_{3}+s_{0} s_{3}-s_{1} p_{2}+s_{2} p_{1}\right)\right],
\end{aligned}
$$

in terms of the quark bilinears Equation (11).

\subsection{Functional Integral and Bosonic Variables}

We proceed to show that the pure fermionic Lagrangian density Equation (2) is dynamically equivalent to a semibosonized Lagrangian containing eight auxiliary boson fields $\sigma=\sigma_{\alpha} \tau_{\alpha}, \pi=\pi_{\alpha} \tau_{\alpha}$ with the following functional integral representation

$$
\begin{gathered}
Z=\int d \sigma_{\alpha} d \pi_{\alpha} d \psi d \bar{\psi} \exp \left(i \int d^{4} x \mathcal{L}^{\prime}\right), \\
\mathcal{L}^{\prime}=\mathcal{L}_{f}+\mathcal{L}_{\pi, \sigma}+\mathcal{L}_{\mathrm{YM}},
\end{gathered}
$$

where $\mathcal{L}_{f}$ involves the fermionic fields

$$
\mathcal{L}_{f}=\bar{\psi}\left(i \gamma^{\mu} \mathcal{D}_{\mu}+\sigma+i \gamma_{5} \pi\right) \psi
$$

and $\mathcal{L}_{\pi, \sigma}$ is a quadratic form involving the spin 0 bosonic fields

$$
\begin{aligned}
\mathcal{L}_{\pi, \sigma}= & -\frac{1}{\bar{g}^{2}}\left[\left(g_{1}+g_{2}\right)\left(\pi_{0}^{2}+\sigma_{i}^{2}\right)+\left(g_{1}-g_{2}\right)\left(\sigma_{0}^{2}+\pi_{i}^{2}\right)\right. \\
& \left.-2 g_{3}\left(\pi_{0} \pi_{3}+\sigma_{0} \sigma_{3}-\sigma_{1} \pi_{2}+\sigma_{2} \pi_{1}\right)\right],
\end{aligned}
$$

with

$$
\bar{g}^{2} \equiv g_{1}^{2}-g_{2}^{2}-g_{3}^{2} \neq 0,
$$

and the covariant derivative for the gauge fields is given by

$$
\mathcal{D}_{\mu} \psi=\left[\partial_{\mu}-i g T_{i} A_{\mu}^{i} P_{L}+i g^{\prime} B_{\mu}\left(T_{3} P_{L}-Q\right)\right] \psi .
$$

The Lagrangian Equation (24) corresponds to a minimal bosonization, [38], see also the discussion in [36], meaning that the number of parameters is kept the same as in Equation (2). The equivalence of the functional integrals involving the Lagrangian densities Equations (2) and (22) is proven, integrating over the auxiliary fields, which can precisely be done since the auxiliary fields are a quadratic form. The dynamical equivalence of both Lagrange densities is guaranteed if Equations (2) and (22) are the same for the solutions given by the Euler-Lagrange equations for the static auxiliary fields $\sigma_{\alpha}, \pi_{\alpha}$

$$
\frac{\partial \mathcal{L}^{\prime}}{\partial \sigma_{\alpha}}=0, \quad \frac{\partial \mathcal{L}^{\prime}}{\partial \pi_{\alpha}}=0
$$

These are readily obtained and establish the following links between the auxiliary coordinates and the bilinears $\bar{\psi} \tau_{\alpha} \psi$ and $\bar{\psi} i \gamma_{5} \tau_{\alpha} \psi$ 


$$
\begin{aligned}
& 2 \sigma_{0}=\left(g_{1}+g_{2}\right) \bar{\psi} \tau_{0} \psi+g_{3} \bar{\psi} \tau_{3} \psi, \\
& 2 \sigma_{1}=\left(g_{1}-g_{2}\right) \bar{\psi} \tau_{1} \psi-g_{3} \bar{\psi} i \gamma_{5} \tau_{2} \psi, \\
& 2 \sigma_{2}=\left(g_{1}-g_{2}\right) \bar{\psi} \tau_{2} \psi+g_{3} \bar{\psi} i \gamma_{5} \tau_{1} \psi, \\
& 2 \sigma_{3}=\left(g_{1}-g_{2}\right) \bar{\psi} \tau_{3} \psi+g_{3} \bar{\psi} \tau_{0} \psi, \\
& 2 \pi_{0}=\left(g_{1}-g_{2}\right) \bar{\psi} i \gamma_{5} \tau_{0} \psi+g_{3} \bar{\psi} i \gamma_{5} \tau_{3} \psi, \\
& 2 \pi_{1}=\left(g_{1}+g_{2}\right) \bar{\psi} i \gamma_{5} \tau_{1} \psi+g_{3} \bar{\psi} \tau_{2} \psi, \\
& 2 \pi_{2}=\left(g_{1}+g_{2}\right) \bar{\psi} i \gamma_{5} \tau_{2} \psi-g_{3} \bar{\psi} \tau_{1} \psi, \\
& 2 \pi_{3}=\left(g_{1}+g_{2}\right) \bar{\psi} i \gamma_{5} \tau_{3} \psi+g_{3} \bar{\psi} i \gamma_{5} \tau_{0} \psi .
\end{aligned}
$$

These expressions show that the quark-antiquark states $\sigma_{0}, \sigma_{3}$ are described by linear combinations of scalar bilinears, and the $\pi_{0}, \pi_{3}$ by a superposition of two pseudoscalars. The other four states involve admixtures of different parities. With these solutions substituted in Equation (22), one gets

$$
\begin{aligned}
& \bar{\psi}\left(\sigma+i \gamma_{5} \pi\right) \psi+\mathcal{L}_{\pi, \sigma}=\frac{1}{2} \bar{\psi}\left(\sigma+i \gamma_{5} \pi\right) \psi \\
& =\frac{g_{1}}{4}\left[\left(\bar{\psi} \tau_{\alpha} \psi\right)^{2}+\left(\bar{\psi} i \gamma_{5} \tau_{\alpha} \psi\right)^{2}\right] \\
& +\frac{g_{2}}{4}\left[\left(\bar{\psi} \tau_{0} \psi\right)^{2}-\left(\bar{\psi} \tau_{i} \psi\right)^{2}-\left(\bar{\psi} i \gamma_{5} \tau_{0} \psi\right)^{2}+\left(\bar{\psi} i \gamma_{5} \tau_{i} \psi\right)^{2}\right] \\
& +\frac{g_{3}}{2}\left[\left(\bar{\psi} \tau_{0} \psi\right)\left(\bar{\psi} \tau_{3} \psi\right)-\left(\bar{\psi} \tau_{1} \psi\right)\left(\bar{\psi} i \gamma_{5} \tau_{2} \psi\right)\right. \\
& \left.\quad+\left(\bar{\psi} \tau_{2} \psi\right)\left(\bar{\psi} i \gamma_{5} \tau_{1} \psi\right)+\left(\bar{\psi} i \gamma_{5} \tau_{0} \psi\right)\left(\bar{\psi} i \gamma_{5} \tau_{3} \psi\right)\right] .
\end{aligned}
$$

The Yukawa form involving the fields $\sigma, \pi, \psi$ is seen to be identical to Equation (20) and proves the equivalence of the Lagrangian density Equations (2) and (22).

\subsection{Symmetry Properties of Composite Fields}

We are now ready to discuss how the eight $\sigma, \pi$ fields relate to two Higgs doublets (see Equations (37), (38) and (44) below)

$$
\Phi_{1}=\left(\begin{array}{c}
\pi_{2}+i \pi_{1} \\
\sigma_{0}-i \pi_{3}
\end{array}\right), \quad \Phi_{2}=\left(\begin{array}{c}
\sigma_{1}-i \sigma_{2} \\
-\sigma_{3}+i \pi_{0}
\end{array}\right)
$$

with $U(1)$ assignment of hypercharge $Y_{L}=1 / 2$, allowing us to connect to standard descriptions of 2HDM and the corresponding classification of symmetries [39-41]. For that, we start from the symmetry transformations of the quark fields underlying the collective $\sigma, \pi$ states in Equations (21) and (22). The infinitesimal $U(2)_{V} \times U(2)_{A}$ transformation for the quark fields is

$$
\delta \psi=i\left(\alpha+\gamma_{5} \beta\right) \psi, \quad \delta \bar{\psi}=i \bar{\psi}\left(-\alpha+\gamma_{5} \beta\right),
$$

where $\alpha=\frac{1}{2} \alpha_{a} \tau_{a}, \beta=\frac{1}{2} \beta_{a} \tau_{a}$, and $\alpha_{a}, \beta_{a}$ are the eight $(a=0,1,2,3)$ infinitesimal parameters of the global transformations of the vector $U(2)_{V}$ and axial-vector $U(2)_{A}$ groups. The Yukawa term $\bar{\psi}\left(\sigma+i \gamma_{5} \pi\right) \psi$ remains invariant under transformations of Equation (31) leading to bosonic fields transforming chirally as

$$
\delta \sigma=i[\alpha, \sigma]+\{\beta, \pi\}, \quad \delta \pi=i[\alpha, \pi]-\{\beta, \sigma\} .
$$

The spin 0 fields are real-valued; thus, their transformations are independent of $\alpha_{0}$. Therefore, we have the $S U(2)_{V} \times U(2)_{A}$ group and use for that

$$
\left[\tau_{i}, \tau_{j}\right]=2 i e_{i j k} \tau_{k}, \quad\left\{\tau_{a}, \tau_{b}\right\}=2 h_{a b c} \tau_{c}
$$


with $i, j, k=1,2,3$ and totally symmetric coefficients $h_{a b c}$ with the non-vanishing components $h_{000}=1, h_{0 i j}=\delta_{i j}$. This yields

$$
\begin{aligned}
& \delta \sigma_{a}=\frac{1}{2} \operatorname{tr}\left(\tau_{a} \delta \sigma\right)=h_{a b c} \beta_{b} \pi_{c}-\delta_{a k} e_{k i j} \alpha_{i} \sigma_{j} \\
& \delta \pi_{a}=\frac{1}{2} \operatorname{tr}\left(\tau_{a} \delta \pi\right)=-h_{a b c} \beta_{b} \sigma_{c}-\delta_{a k} e_{k i j} \alpha_{i} \pi_{j}
\end{aligned}
$$

or, in components

$$
\begin{aligned}
& \delta \sigma_{0}=\beta_{a} \pi_{a}, \quad \delta \sigma_{i}=-e_{i j k} \alpha_{j} \sigma_{k}+\beta_{0} \pi_{i}+\beta_{i} \pi_{0}, \\
& \delta \pi_{0}=-\beta_{a} \sigma_{a}, \quad \delta \pi_{i}=-e_{i j k} \alpha_{j} \pi_{k}-\beta_{0} \sigma_{i}-\beta_{i} \sigma_{0},
\end{aligned}
$$

one obtains then the action of $S U(2)_{V} \times U(2)_{A}$ on the complex fields that enter in the classification Equation (30)

$$
\begin{aligned}
\delta\left(\sigma_{0}-i \pi_{3}\right)= & i\left[\alpha_{1} \pi_{2}-\alpha_{2} \pi_{1}+\beta_{0} \sigma_{3}+\beta_{3} \sigma_{0}-i \beta_{a} \pi_{a}\right], \\
\delta\left(\pi_{2}+i \pi_{1}\right)= & i\left[-\left(\alpha_{1}-i \alpha_{2}\right) i \pi_{3}-\left(\beta_{1}-i \beta_{2}\right) \sigma_{0}\right. \\
& \left.+\alpha_{3}\left(\pi_{2}+i \pi_{1}\right)-\beta_{0}\left(\sigma_{1}-i \sigma_{2}\right)\right] \\
\delta\left(i \pi_{0}-\sigma_{3}\right)= & \alpha_{1} \sigma_{2}-\alpha_{2} \sigma_{1}-\beta_{0} \pi_{3}-\beta_{3} \pi_{0}-i \beta_{a} \sigma_{a}, \\
\delta\left(\sigma_{1}-i \sigma_{2}\right)= & i\left[\alpha_{3}\left(\sigma_{1}-i \sigma_{2}\right)-\left(\alpha_{1}-i \alpha_{2}\right) \sigma_{3}\right. \\
- & \left.\beta_{0}\left(\pi_{2}+i \pi_{1}\right)-\pi_{0}\left(\beta_{2}+i \beta_{1}\right)\right] .
\end{aligned}
$$

These expressions can now be used to finally obtain the transformation rules for the chiral $S U(2)_{L} \times U(1)_{A}$ group, extracting the correct set of infinitesimal parameters as follows. Taking $\alpha_{L}=\alpha-\beta, \alpha_{R}=\alpha+\beta$ that relate $U(2)_{V} \times U(2)_{A}$ to $U(2)_{L} \times U(2)_{R}$, one obtains (with $\alpha_{0}=0, \beta_{0} \neq 0$ and $\alpha_{i}+\beta_{i}=0$ ) four parameters, $\alpha_{i}=-\beta_{i} \equiv \omega_{i} / 2$ and $\beta_{0}$, to describe the infinitesimal transformations of $S U(2)_{L} \times U(1)_{A}$. Rewriting Equation (36) with these parameters, one has

$$
\begin{aligned}
\delta\left(\sigma_{0}-i \pi_{3}\right) & =\frac{i}{2}\left[\left(\omega_{1}+i \omega_{2}\right)\left(\pi_{2}+i \pi_{1}\right)-\omega_{3}\left(\sigma_{0}-i \pi_{3}\right)\right] \\
& -i \beta_{0}\left(i \pi_{0}-\sigma_{3}\right), \\
\delta\left(\pi_{2}+i \pi_{1}\right) & =\frac{i}{2}\left[\left(\omega_{1}-i \omega_{2}\right)\left(\sigma_{0}-i \pi_{3}\right)+\omega_{3}\left(\pi_{2}+i \pi_{1}\right)\right] \\
& -i \beta_{0}\left(\sigma_{1}-i \sigma_{2}\right), \\
\delta\left(i \pi_{0}-\sigma_{3}\right) & =\frac{i}{2}\left[\left(\omega_{1}+i \omega_{2}\right)\left(\sigma_{1}-i \sigma_{2}\right)-\omega_{3}\left(i \pi_{0}-\sigma_{3}\right)\right] \\
& -i \beta_{0}\left(\sigma_{0}-i \pi_{3}\right), \\
\delta\left(\sigma_{1}-i \sigma_{2}\right) & =\frac{i}{2}\left[\left(\omega_{1}-i \omega_{2}\right)\left(i \pi_{0}-\sigma_{3}\right)+\omega_{3}\left(\sigma_{1}-i \sigma_{2}\right)\right] \\
& -i \beta_{0}\left(\pi_{2}+i \pi_{1}\right) .
\end{aligned}
$$

These formulas show that $\Phi_{1}$ and $\Phi_{2}$ in Equation (30) behave like fundamental $S U(2)_{L}$-doublets

$$
\delta \Phi_{1,2}=i \omega_{i} \frac{\tau_{i}}{2} \Phi_{1,2}-i \beta_{0} \Phi_{2,1}
$$

Furthermore, the non-vanishing of the $\beta_{0}$ parameter related to the $U(1)_{A}$ transformation leads to a mixing between the two $\Phi_{i}$ doublets. It is possible to change the basis to

$$
\Phi_{1}=\frac{1}{\sqrt{2}}\left(\Phi_{1}^{\prime}+\Phi_{2}^{\prime}\right), \quad \Phi_{2}=\frac{1}{\sqrt{2}}\left(\Phi_{2}^{\prime}-\Phi_{1}^{\prime}\right),
$$


where the axial transformation does not mix the doublets, but leaves its signature in the sign of $\beta_{0}$

$$
\delta \Phi_{1,2}^{\prime}=i \omega_{i} \frac{\tau_{i}}{2} \Phi_{1,2}^{\prime} \pm i \beta_{0} \Phi_{1,2}^{\prime}
$$

This $U(1)$ symmetry (suitably extended to the quark sector) was first introduced by Peccei and Quinn $[42,43]$ in connection with the strong-CP problem.

\subsection{Quark Content of Higgs Doublets}

From Equation (28), one can also obtain the explicit quark flavor content of the doublet members

$$
\begin{aligned}
& \sigma_{0}-i \pi_{3}=\left(g_{1}+g_{2}+g_{3}\right) \bar{t}_{L} t_{R}+\left(g_{1}+g_{2}-g_{3}\right) \bar{b}_{R} b_{L} \\
& \pi_{2}+i \pi_{1}=\left(g_{1}+g_{2}-g_{3}\right) \bar{b}_{R} t_{L}-\left(g_{1}+g_{2}+g_{3}\right) \bar{b}_{L} t_{R} \\
& i \pi_{0}-\sigma_{3}=\left(g_{1}-g_{2}-g_{3}\right) \bar{b}_{R} b_{L}-\left(g_{1}-g_{2}+g_{3}\right) \bar{t}_{L} t_{R} \\
& \sigma_{1}-i \sigma_{2}=\left(g_{1}-g_{2}-g_{3}\right) \bar{b}_{R} t_{L}+\left(g_{1}-g_{2}+g_{3}\right) \bar{b}_{L} t_{R} .
\end{aligned}
$$

The distinguishing feature of these doublet members written in terms of the quark content of collective states $\sigma, \pi$ is that they involve the couplings $g_{i}$. If $g_{3}=0$, one can isolate two doublets, which we denote by $\Phi_{1}^{0}, \Phi_{2}^{0}$

$$
\begin{aligned}
& \Phi_{1}^{0}=\left(\begin{array}{l}
\bar{b}_{R} t_{L}-\bar{b}_{L} t_{R} \\
\bar{b}_{R} b_{L}+\bar{t}_{L} t_{R}
\end{array}\right), \\
& \Phi_{2}^{0}=\left(\begin{array}{l}
\bar{b}_{R} t_{L}+\bar{b}_{L} t_{R} \\
\bar{b}_{R} b_{L}-\bar{t}_{L} t_{R}
\end{array}\right) .
\end{aligned}
$$

Both doublets possess $U(1)$ hypercharge $Y_{L}=Q-T_{3}=1 / 2$; their upper components have positive charge $Q=1$ and isopin projection $T_{3}=1 / 2$, whereas the lower components carry $Q=0, T_{3}=-1 / 2$. In terms of these, one obtains

$$
\left(\begin{array}{l}
\Phi_{1} \\
\Phi_{2}
\end{array}\right)=\left(\begin{array}{cc}
\left(g_{1}+g_{2}\right) & -g_{3} \\
-g_{3} & \left(g_{1}-g_{2}\right)
\end{array}\right)\left(\begin{array}{l}
\Phi_{1}^{0} \\
\Phi_{2}^{0}
\end{array}\right) .
$$

In this form, the doublets $\Phi_{1}, \Phi_{2}$ represent an admixture of the quark doublets $\Phi_{1}^{0}, \Phi_{2}^{0}$, which carry different weights $(g 1 \pm g 2)$ and are coupled via the $g_{3}$ interaction.

\section{Schwinger-DeWitt Approach}

Having shown that the path integral representation Equation (21) with the auxiliary bosonic variables $\sigma, \pi$ conduces to two Higgs doublets with the required symmetry and definite quark content, we proceed to integrate out the short-distance components of the quark fields $\psi \rightarrow \psi+\psi_{s d}$. For that, Wilson's method [44] is used, taking into consideration the analyses of [19], from which one deduces that upon integration, the auxiliary scalar fields $\sigma$ and $\pi$ acquire gauge-invariant kinetic terms and quartic interactions in the effective action at scales $\mu$ below the cutoff $\Lambda$. Here we derive the induced kinetic and quartic interactions for Lagrangian Equation (22) using the Schwinger-DeWitt technique [45-48]. Although the Lagrangian density Equation (24) requires a diagonalization of the quadratic form, we postpone it to after having obtained the induced effective contribution to the Lagrangian, which is also non-diagonal. We start by considering the functional integral associated to the four fermion vertices that develop at the scale $\Lambda$, Equation (23), with the fermionic degrees of freedom divided into short-distance components $\psi_{s d}$ and remaining components $\psi_{r}$ which, for notational simplicity, we relabel as $\psi$

$$
Z_{f, \Lambda}=\int d \psi d \bar{\psi} \exp \left(i \int d^{4} x \mathcal{L}_{f}\right) Z_{s d, \mu}
$$




$$
Z_{s d, \mu}=\int d \psi_{s d} d \bar{\psi}_{s d} \exp \left(i \int d^{4} x \mathcal{L}_{f, s d}\right) .
$$

The integration over the high-frequency components is performed using $Z_{s d, \mu}$ in the one-loop fermion approximation, from the large-scale $\Lambda$ down to the scale $\mu$, making sure that at $\mu=\Lambda$ one recovers the functional in terms of the initial four-fermion configuration. We need the real part of the action which is obtained from the inverse of the fermionic propagator containing the background fields $\sigma, \pi$ and gauge fields, and is formally given by its functional determinant. Since the Euclidean propagator is well-defined as the kernel of the inverse of an elliptic operator $\Delta$ for $x \neq y$

$$
\begin{aligned}
G(x, y) & =\left\langle x\left|\Delta^{-1}\right| y\right\rangle=\int_{0}^{\infty} d t\left\langle x\left|\mathrm{e}^{-t \Delta}\right| y\right\rangle \\
& =\int_{0}^{\infty} d t \mathcal{G}(x, y, t),
\end{aligned}
$$

the Schwinger or proper time representation for the propagator $G(x, y)$ is given in four space-time dimensions as

$$
G(x, y)=\int_{0}^{\infty} \frac{d t}{(4 \pi t)^{2}} \mathrm{e}^{-\frac{|x-y|^{2}}{4 t}} F(x, y, t),
$$

where $F$ parametrizes deviations from free propagation $\Delta_{0}=-\partial^{2}$. In this form, the infrared content of $G$ is controlled by the behaviour of $F(x, y, t)$ at large values of $t$, while the short-distance behavior of $F(x, y, t)$ is given at small values of the proper time $t$ by the asymptotic expansion

$$
F \sim \sum_{n=0}^{\infty} a_{n}(x, y) t^{n}
$$

where $a_{n}(x, y)$ are the Seeley-DeWitt coefficients. Comparing Equations (47) and (48) yields in the coincidence limit the $t \rightarrow 0$ behavior for

$$
\mathcal{G}(x, x, t) \sim \frac{1}{(4 \pi t)^{2}} \sum_{n=0}^{\infty} a_{n} t^{n},
$$

where $a_{n} \equiv a_{n}(x, x)$. In the present case, the ellyptic operator is given by $\Delta=D_{E}^{\dagger} D_{E}$, and the Euclidean effective action is obtained as the short-distance contribution to the functional trace at each proper time considered, involving the scales

$$
\operatorname{Re} S_{E}=\frac{1}{2} \ln \left|\operatorname{det} D_{E}^{\dagger} D_{E}\right|=-\frac{1}{2} \int_{0}^{\infty} \frac{d t}{t} \rho_{\mu}^{\Lambda}(t) \operatorname{Tr}\left(e^{-t D_{E}^{\dagger} D_{E}}\right)_{s d},
$$

and one identifies $\operatorname{Tr}\left(e^{-t D_{E}^{\dagger} D_{E}}\right)_{s d}=\int d^{4} x \mathcal{G}(x, x, t)$. The quantities in Equation (51) are given as

$$
D_{E}^{\dagger} D_{E}=-\mathrm{d}_{\alpha}^{2}+Y
$$

where $D_{E}$ is the fermionic inverse propagator in the presence of background fields as obtained in Equation (23),

$$
D_{E}=\left(i \gamma_{\alpha} \mathrm{d}_{\alpha}+\sigma+i \gamma_{5} \pi\right)
$$

and $\mathrm{d}_{\alpha}=\partial_{\alpha}+i \Gamma_{\alpha}$ is the covariant derivative ( $\Gamma_{\alpha}$ is defined in Equation (59) in the Minkowski space); the symbol $E$ denotes Euclidean space. The resulting dependence in the collective fields is given in $Y$ in Equation (57). The kernel $\rho_{\mu}^{\Lambda}(t)$ restricts the propertime integration to the interval involving the pertinent scales, $\rho_{\mu}^{\Lambda}(t)=\theta\left(1-t \mu^{2}\right) \theta\left(t \Lambda^{2}-1\right)$. 
One finally obtains that the induced terms due to the integration over the high-frequency modes of the quark components contribute to the real part of the effective action in the form of the asymptotic series in proper time

$$
\operatorname{Re} S_{E}=-\frac{1}{2} \int \frac{d t}{t^{3}} \int d^{4} x_{E} \frac{1}{(4 \pi)^{2}} \sum_{n=0}^{\infty} \rho_{\mu}^{\Lambda}(t) t^{n} \operatorname{tr}\left(a_{n}^{E}\right),
$$

where the Seeley-DeWitt coefficients $a_{n}^{E}$ depend on fields and encode the high-energy behavior of the theory. We only need to consider the coefficients $a_{1}^{E}=-Y, a_{2}^{E}=Y^{2} / 2-$ $F_{\mu \nu}^{2} / 12$, since they pertains to integrals that diverge in proper time, providing for dominant contributions. We also note that $a_{0}^{E}$ does not have fields, and is irrelevant in the present case. The integrals considered are denoted as $C_{1}$ and $C_{2}$ and involve two scale parameters, $\Lambda$ and $\mu$

$$
\begin{aligned}
& C_{1}=\int_{1 / \Lambda^{2}}^{1 / \mu^{2}} \frac{d t}{t^{2}}=\Lambda^{2}-\mu^{2}, \\
& C_{2}=\int_{1 / \Lambda^{2}}^{1 / \mu^{2}} \frac{d t}{t}=\ln \frac{\Lambda^{2}}{\mu^{2}} .
\end{aligned}
$$

Here, $\Lambda$ determines the high energy scale at which effective four-quark interactions of Equation (9) set in. The low-energy scale parameter $\mu$ relates to the induced one-loop proper-time contributions. For $\mu=\Lambda$, all contributions vanish, as required by [19]. The functions $Y$ are determined to be

$$
\begin{aligned}
Y & =\sigma^{2}+\pi^{2}+i \gamma_{5}[\sigma, \pi]-i \nabla_{\mu}\left(\gamma^{\mu} \sigma+i \gamma^{\mu} \gamma_{5} \pi\right) \\
& -\frac{i}{4}\left[\gamma_{\mu}, \gamma_{\nu}\right] F^{\mu v}, \\
F_{\mu v} & =\partial_{\mu} \Gamma_{\nu}-\partial_{\nu} \Gamma_{\mu}-i\left[\Gamma_{\mu}, \Gamma_{v}\right] \\
\Gamma_{\mu} & =g T_{i} A_{\mu}^{i} P_{L}-g^{\prime} B_{\mu}\left(P_{L} T_{3}-Q\right),
\end{aligned}
$$

where the conversion to Minkowski space has already been effected [49]. The covariant derivatives depend on $\gamma_{5}$ and are given by

$$
\begin{aligned}
& \nabla_{\mu}\left(\gamma^{\mu} \sigma\right)=\partial_{\mu}\left(\gamma^{\mu} \sigma\right)-i\left[\Gamma_{\mu}, \gamma^{\mu} \sigma\right], \\
& \nabla_{\mu}\left(i \gamma^{\mu} \gamma_{5} \pi\right)=\partial_{\mu}\left(i \gamma^{\mu} \gamma_{5} \pi\right)-i\left[\Gamma_{\mu}, i \gamma^{\mu} \gamma_{5} \pi\right] .
\end{aligned}
$$

The result of the proper-time integration can be cast as

$$
\Delta \mathcal{L}_{s d}=-\frac{1}{32 \pi^{2}}\left[C_{1} \operatorname{tr}(-Y)+C_{2} \operatorname{tr}\left(\frac{Y^{2}}{2}-\frac{F_{\mu v}^{2}}{12}\right)\right],
$$

representing the anticipated additional induced gauge invariant $S U(2)_{L} \times U(1)_{R}$ contribution to the Lagrangian at low energy scales $\mu \ll \Lambda$

$$
\tilde{\mathcal{L}}=\mathcal{L}^{\prime}+\Delta \mathcal{L}_{s d}
$$

The traces are to be taken in isospin, Dirac, and color spaces. 


\section{Higgs Sector}

The quadratic and quartic powers in the bosonic fields in Equation (63) lead to the Higgs potential

$$
\begin{aligned}
& V_{H}=-\mathcal{L}_{\pi, \sigma}-\bar{C}_{1}\left(\sigma_{\alpha}^{2}+\pi_{\alpha}^{2}\right)+2 \bar{C}_{2}\left[\frac{1}{4}\left(\sigma_{\alpha}^{2}+\pi_{\alpha}^{2}\right)^{2}\right. \\
& \left.+\left(\sigma_{0}^{2}+\pi_{i}^{2}\right)\left(\sigma_{i}^{2}+\pi_{0}^{2}\right)-\left(\sigma_{0} \pi_{0}-\sigma_{i} \pi_{i}\right)^{2}\right]
\end{aligned}
$$

where $\bar{C}_{1,2} \equiv N_{c} C_{1,2} /\left(4 \pi^{2}\right)$. In terms of the two Higgs electroweak doublets (30), one gets

$$
\begin{aligned}
& V_{H}=-\bar{C}_{1}\left(\Phi_{1}^{\dagger} \Phi_{1}+\Phi_{2}^{\dagger} \Phi_{2}\right)+2 \bar{C}_{2} \\
& {\left[\frac{1}{4}\left(\Phi_{1}^{\dagger} \Phi_{1}+\Phi_{2}^{\dagger} \Phi_{2}\right)^{2}+\left(\Phi_{1}^{\dagger} \Phi_{1}\right)\left(\Phi_{2}^{\dagger} \Phi_{2}\right)-\left(\operatorname{Im}\left(\Phi_{1}^{\dagger} \Phi_{2}\right)\right)^{2}\right]} \\
& +\frac{1}{\bar{g}^{2}}\left[\left(g_{1}-g_{2}\right) \Phi_{1}^{\dagger} \Phi_{1}+\left(g_{1}+g_{2}\right) \Phi_{2}^{\dagger} \Phi_{2}+2 g_{3} \operatorname{Re}\left(\Phi_{1}^{\dagger} \Phi_{2}\right)\right] .
\end{aligned}
$$

At this stage, one can compare with [39] and identify in their notation three mass terms and four real-valued and independent quartic couplings

$$
\begin{aligned}
& m_{11}^{2}=\frac{g_{1}-g_{2}}{\bar{g}^{2}}-\bar{C}_{1}, \quad m_{22}^{2}=\frac{g_{1}+g_{2}}{\bar{g}^{2}}-\bar{C}_{1}, \quad m_{12}^{2}=-\frac{g_{3}}{\bar{g}^{2}}, \\
& \lambda_{1}=\lambda_{2}=\frac{1}{3} \lambda_{3}=-\lambda_{4}=\lambda_{5}=\bar{C}_{2},
\end{aligned}
$$

instead of eight possible in the general case.

\subsection{Dynamical Symmetry Breaking and Bottom Quark Mass Catalysis}

The model considered displays of critical behavior, giving origin to the quark masses. This occurs when the neutral scalar fields acquire non-vanishing vacuum expectation values $\left\langle\sigma_{0}\right\rangle=-m_{0},\left\langle\sigma_{3}\right\rangle=-m_{3}$. These scalar fields must be redefined such that the minimum configuration of the potential in the new variables correspond to vanishing of their vacuum expectation values. This is achieved by shifting

$$
\sigma_{0} \rightarrow \sigma_{0}-m_{0}, \quad \sigma_{3} \rightarrow \sigma_{3}-m_{3} .
$$

The gap equations then result in the removal from the potential $V_{H}$ of linear terms in these new fields

$$
\begin{aligned}
& m_{0}\left(g_{1}-g_{2}\right)-m_{3} g_{3}=\bar{g}^{2} m_{0}\left[\bar{C}_{1}-\left(m_{0}^{2}+3 m_{3}^{2}\right) \bar{C}_{2}\right], \\
& m_{3}\left(g_{1}+g_{2}\right)-m_{0} g_{3}=\bar{g}^{2} m_{3}\left[\bar{C}_{1}-\left(m_{3}^{2}+3 m_{0}^{2}\right) \bar{C}_{2}\right] .
\end{aligned}
$$

One can use Equation (28) to analyse the possible solutions of the gap equations in terms of the quark condensates $\langle\bar{b} b\rangle,\langle\bar{t} t\rangle$

$$
\begin{aligned}
& 2\left\langle\sigma_{0}\right\rangle=\left(g_{1}+g_{2}+g_{3}\right)\langle\bar{t} t\rangle+\left(g_{1}+g_{2}-g_{3}\right)\langle\bar{b} b\rangle, \\
& 2\left\langle\sigma_{3}\right\rangle=\left(g_{1}-g_{2}+g_{3}\right)\langle\bar{t} t\rangle-\left(g_{1}-g_{2}-g_{3}\right)\langle\bar{b} b\rangle .
\end{aligned}
$$

Using the top and bottom quark masses $m_{t}=m_{0}+m_{3}, m_{b}=m_{0}-m_{3}$ they read

$$
\begin{aligned}
& m_{t}=-\left(g_{1}+g_{3}\right)\langle\bar{t} t\rangle-g_{2}\langle\bar{b} b\rangle, \\
& m_{b}=-g_{2}\langle\bar{t} t\rangle-\left(g_{1}-g_{3}\right)\langle\bar{b} b\rangle .
\end{aligned}
$$

These show that the absence of the bottom quark condensate does not necessarily mean that the bottom quark mass is zero: in the case that $g_{2} \neq 0$, that is, when the $U(1)_{A}$ symmetry is violated, the top quark condensate also provides mass to the bottom quark. If 
instead $g_{2}=0$, but $g_{3} \neq 0$, finite quark masses are always produced together with their respective condensates. Both possibilities comply with solutions of the gap equations, which we address now. Using the physical $m_{b}, m_{t}$ quark masses, one can represent the gap equations as

$$
\begin{aligned}
& \left(g_{1}-g_{3}\right) m_{t}-g_{2} m_{b}=\bar{g}^{2} m_{t}\left(\bar{C}_{1}-m_{t}^{2} \bar{C}_{2}\right), \\
& \left(g_{1}+g_{3}\right) m_{b}-g_{2} m_{t}=\bar{g}^{2} m_{b}\left(\bar{C}_{1}-m_{b}^{2} \bar{C}_{2}\right) .
\end{aligned}
$$

The trivial solution given by zero quark masses occurs at $\mu=\Lambda$ and is the only solution since $\bar{g}^{2} \neq 0$. At scales $\mu<\Lambda$, the equations can have non-trivial solutions according to the values of the couplings.

Let us first consider the case $g_{2}=g_{3}=0$. As mentioned before, this corresponds to the original NJL model. The critical point for the transition from the symmetric Wigner-Weyl phase to the Nambu-Goldstone phase occurs when the coupling $g_{1}$ surpasses the critical value $g_{c}$.

Next, take the case with $g_{2}=0$ and both $g_{1}, g_{3}$ different from 0 . Then the system of Equations (72) and (73) decouples in the quark masses

$$
\begin{aligned}
& m_{b}^{2} \bar{C}_{2}=\bar{C}_{1}-\frac{1}{g_{1}-g_{3}}, \\
& m_{t}^{2} \bar{C}_{2}=\bar{C}_{1}-\frac{1}{g_{1}+g_{3}} .
\end{aligned}
$$

Thus, one sees that solutions with finite $m_{b}$ can occur even for $g_{2}=0$, if the combination $g_{1}-g_{3}>g_{c}=4 \pi^{2} / N_{c} \Lambda^{2}$. This result is in contrast with the model [22], where a finite $m_{b}$ can be generated if, and only if $g_{2} \neq 0$, so when the $U(1)_{A}$ symmetry is violated.

Finally, we discuss what is meant by catalysis of the $\langle\bar{b} b\rangle$ condensate. If the condition $g_{1}>g_{c}$ holds, which we saw was necessary to generate the quark masses, then an arbitrarily small value of the coupling $g_{3}$ will suffice to create the bottom condensate, thus acting as a catalyst (see also [50]). This phenomenon occurs because $g_{3}$ is the source of isospin violation in the model.

\subsection{Diagonalization}

As mentioned earlier, the quadratic part of $V_{H}$ in the Lagrangian Equation (65) must be diagonalized. This is a convenient place to do it, as the gap equations carry information on the two diagonalization angles: the angle $\theta$ that diagonalizes the charged sector, and $\theta^{\prime}$ for the neutral one. They are related to each other and to ratios involving the quark masses and the couplings $g_{3}, g_{2}$ through

$$
\tan \theta=\frac{m_{3}}{m_{0}}, \quad \tan 2 \theta^{\prime}=3 \tan 2 \theta-2 \frac{g_{3}}{g_{2}} .
$$

Please recall that the states in Equation (65) are defined prior to the redefinition of the scalar neutral fields that acquire vacuum expectation values. We return to this point in a moment.

In Appendix A are given all details needed to obtain the mass eigenstates

$$
\begin{aligned}
& \Phi_{1}=H_{1} \cos \theta+H_{2} \sin \theta=\frac{1}{m}\left(m_{0} H_{1}+m_{3} H_{2}\right), \\
& \Phi_{2}=H_{2} \cos \theta-H_{1} \sin \theta=\frac{1}{m}\left(m_{0} H_{2}-m_{3} H_{1}\right),
\end{aligned}
$$

where

$$
H_{1}=\left(\begin{array}{c}
\tilde{\pi}_{2}+i \tilde{\pi}_{1} \\
\sigma_{0}^{\prime}-m-i \tilde{\pi}_{3}
\end{array}\right), \quad H_{2}=\left(\begin{array}{c}
\tilde{\sigma}_{1}-i \tilde{\sigma}_{2} \\
-\sigma_{3}^{\prime}+i \tilde{\pi}_{0}
\end{array}\right) .
$$


Here, $m=\sqrt{m_{0}^{2}+m_{3}^{2}} \simeq m_{t} / \sqrt{2}$. The notation is as follows. The fields with a tilde result from the orthogonal transformations involving the angles $\theta^{\prime} \neq \theta$. Now, since $\sigma_{0}$ and $\sigma_{3}$ are subject to the shifts of Equation (67), this translates to the transformation with the angle $\alpha=\theta-\theta^{\prime}$ to the final primed variables

$$
\begin{aligned}
& \sigma_{0}^{\prime}=\tilde{\sigma}_{0} \cos \alpha+\tilde{\sigma}_{3} \sin \alpha, \\
& \sigma_{3}^{\prime}=\tilde{\sigma}_{3} \cos \alpha-\tilde{\sigma}_{0} \sin \alpha .
\end{aligned}
$$

The states $H_{1}, H_{2}$ build the so-called Higgs basis. A known property of this basis is that only $H_{1}$ acquires a finite vacuum expectation value [39], here given by $\left\langle H_{1}\right\rangle=(0,-m)$.

A final step must still be undertaken. Until now, the induced kinetic terms of the spin 0 bosonic fields were not commented on. They do not have the canonical form. To achieve this, one must normalize the fields $\tilde{\pi}_{\alpha}=\phi_{\alpha} / \sqrt{\bar{C}_{2}}$, and $\tilde{\sigma}_{\alpha}=\chi_{\alpha} / \sqrt{\bar{C}_{2}}$ [36]. In terms of the redefined (physical) fields, one obtains

$$
\begin{aligned}
& H_{1}=\frac{1}{\sqrt{\bar{C}_{2}}}\left(\begin{array}{c}
\phi_{2}+i \phi_{1} \\
\chi_{0}^{\prime}-\sqrt{\bar{C}_{2}} m-i \phi_{3}
\end{array}\right), \\
& H_{2}=\frac{1}{\sqrt{\bar{C}_{2}}}\left(\begin{array}{c}
\chi_{1}-i \chi_{2} \\
-\chi_{3}^{\prime}+i \phi_{0}
\end{array}\right), \quad\left(\begin{array}{l}
\chi_{0}^{\prime} \\
\chi_{3}^{\prime}
\end{array}\right)=R(\alpha)\left(\begin{array}{l}
\chi_{0} \\
\chi_{3}
\end{array}\right),
\end{aligned}
$$

$R(\alpha)$ being a standard $S O(2)$ rotation.

At this point, we remark that the gap equations can be expressed in terms of $\theta$

$$
\begin{aligned}
& \bar{g}^{2} \bar{C}_{1}=g_{1}-\frac{2 g_{2}}{\cos 2 \theta}+\frac{g_{3}}{\sin 2 \theta}, \\
& \bar{g}^{2} m^{2} \bar{C}_{2}=\frac{g_{3}}{\sin 2 \theta}-\frac{g_{2}}{\cos 2 \theta}
\end{aligned}
$$

which turns out to be helpful to link the states in the spectrum. Before addressing the spectrum in the next section, we give in the following two paragraphs the result for the interaction potential of the Higgs fields, as well as the Yukawa terms (for details, see [36]).

4.3. Higgs Doublets Cubic and Quartic Interactions

Defining

$$
\tilde{H}_{1}=\left(\begin{array}{c}
\phi_{2}+i \phi_{1} \\
\chi_{0}^{\prime}-i \phi_{3}
\end{array}\right), \quad \tilde{H}_{2}=\left(\begin{array}{c}
\chi_{1}-i \chi_{2} \\
-\chi_{3}^{\prime}+i \phi_{0}
\end{array}\right)=\sqrt{\bar{C}_{2}} H_{2}
$$

the interaction potential takes the form

$$
\begin{aligned}
V_{H}^{i n t} & =\frac{2}{\bar{C}_{2}}\left[\frac{1}{4}\left(\tilde{H}_{1}^{\dagger} \tilde{H}_{1}+\tilde{H}_{2}^{\dagger} \tilde{H}_{2}\right)^{2}+\left(\tilde{H}_{1}^{\dagger} \tilde{H}_{1}\right)\left(\tilde{H}_{2}^{\dagger} \tilde{H}_{2}\right)\right. \\
& \left.-\left(\operatorname{Im}\left(\tilde{H}_{1}^{\dagger} \tilde{H}_{2}\right)\right)^{2}\right]-\frac{2 m}{\sqrt{\bar{C}_{2}}}\left(\tilde{H}_{1}^{\dagger} \tilde{H}_{1}+\tilde{H}_{2}^{\dagger} \tilde{H}_{2}\right) \chi_{0}^{\prime} \\
& -\frac{4 m_{0}}{\sqrt{\bar{C}_{2}}}\left(\cos \theta \chi_{0}^{\prime}-\sin \theta \chi_{3}^{\prime}\right) \tilde{H}_{2}^{\dagger} \tilde{H}_{2}+\frac{4 m}{\sqrt{\bar{C}_{2}}} \phi_{0} \operatorname{Im}\left(\tilde{H}_{1}^{\dagger} \tilde{H}_{2}\right) \\
& -\frac{4 m_{3}}{\sqrt{\bar{C}_{2}}}\left(\cos \theta \chi_{3}^{\prime}+\sin \theta \chi_{0}^{\prime}\right) \tilde{H}_{1}^{\dagger} \tilde{H}_{1} .
\end{aligned}
$$




\subsection{Yukawa Couplings}

Lagrangian Equation (63) contains the Yukawa terms

$$
\begin{aligned}
& \mathcal{L}_{Y}=\bar{\psi}\left(\sigma+i \gamma_{5} \pi\right) \psi=\bar{\psi}_{L}(\sigma+i \pi) \psi_{R}+\bar{\psi}_{R}(\sigma-i \pi) \psi_{L} \\
& =\bar{t}_{L} t_{R}\left(\sigma_{0}+\sigma_{3}+i \pi_{0}+i \pi_{3}\right)+\bar{t}_{L} b_{R}\left(\sigma_{1}-i \sigma_{2}+i \pi_{1}+\pi_{2}\right) \\
& +\bar{b}_{L} b_{R}\left(\sigma_{0}-\sigma_{3}+i \pi_{0}-i \pi_{3}\right)+\bar{b}_{L} t_{R}\left(\sigma_{1}+i \sigma_{2}+i \pi_{1}-\pi_{2}\right) \\
& + \text { h.c. }
\end{aligned}
$$

The conversion to the physical fields results in

$$
\begin{aligned}
\mathcal{L}_{Y}= & -\bar{\psi} M \psi+\lambda_{b} \bar{\psi}_{L} \tilde{H}_{1} b_{R}+\lambda_{t} \bar{\psi}_{L}^{a} e_{a b} \tilde{H}_{1 b}^{*} t_{R} \\
& +\lambda_{t} \bar{\psi}_{L} \tilde{H}_{2} b_{R}-\lambda_{b} \bar{\psi}_{L}^{a} e_{a b} \tilde{H}_{2 b}^{*} t_{R}+\text { h.c. },
\end{aligned}
$$

where $M=\operatorname{diag}\left(m_{t}, m_{b}\right), e_{a b}$ is the antisymmetric tensor with $e_{12}=-e_{21}=1$ and

$$
\lambda_{t}=\frac{m_{t}}{m \sqrt{\bar{C}_{2}}} \equiv \frac{y_{t}}{\sqrt{2}}, \quad \lambda_{b}=\frac{m_{b}}{m \sqrt{\bar{C}_{2}}} \equiv \frac{y_{b}}{\sqrt{2}} .
$$

\section{The MTY Two-Higgs-Doublet Spectrum and Nambu Sum Rule}

We proceed to analyse the Higgs masses that stem from Equations (65) and (A14) (see also [51]). The mass eigenstates are expressed by $\chi_{\alpha}$ and $\phi_{\alpha}$ with squared-values

$$
\begin{aligned}
& m_{\chi_{0}}^{2}=4 m^{2}+\frac{2 g_{2}}{\bar{g}^{2} \bar{C}_{2}}\left(\frac{1}{\cos 2 \theta}-\frac{1}{\cos 2 \theta^{\prime}}\right) \\
& m_{\chi_{3}}^{2}=4 m^{2}+\frac{2 g_{2}}{\bar{g}^{2} \bar{C}_{2}}\left(\frac{1}{\cos 2 \theta}+\frac{1}{\cos 2 \theta^{\prime}}\right) \\
& m_{\phi_{0}}^{2}=\frac{4 g_{2}}{\bar{g}^{2} \bar{C}_{2} \cos 2 \theta} \\
& m_{\chi^{ \pm}}^{2}=\frac{4 g_{3}}{\bar{g}^{2} \bar{C}_{2} \sin 2 \theta} \\
& m_{\phi_{i}}^{2}=0 .
\end{aligned}
$$

Out of the eight spinless eigenstates, the last three $\phi_{i}$, are Goldstone modes that are absorbed by gauge fields through the Higgs mechanism. The remaining eigenstates satisfy the sum rule

$$
\begin{aligned}
& m_{\chi_{0}}^{2}+m_{\chi_{3}}^{2}+m_{\phi_{0}}^{2}=\frac{8 g_{3}}{\bar{g}^{2} \bar{C}_{2} \sin 2 \theta}, \\
& m_{\chi^{+}}^{2}+m_{\chi^{-}}^{2}=\frac{8 g_{3}}{\bar{g}^{2} \bar{C}_{2} \sin 2 \theta},
\end{aligned}
$$

where we have used Equation (85). However, although the sum of the squared masses of the neutral and charged modes results in the same expression, it differs from the $4 m_{t}^{2}$ value required by the Nambu sum rule. Moreover, the first expression contains the contributions of three states, instead of two Nambu partners. Let us shed some light on such peculiarities. From the mass formulas (91)-(95), we have

$$
\begin{aligned}
& m_{\chi_{0}}^{2}+m_{\chi_{3}}^{2}=m_{\phi_{0}}^{2}+4\left(m_{t}^{2}+m_{b}^{2}\right), \\
& m_{\chi^{+}}^{2}+m_{\chi^{-}}^{2}=2 m_{\phi_{0}}^{2}+4\left(m_{t}^{2}+m_{b}^{2}\right),
\end{aligned}
$$

which points out the non-zero mass of the $\phi_{0}$ meson as the origin of the Nambu sum rule violation. When there is no interaction in the coupling constant $g_{2}$, the model has an additional $U(1)_{A}$ global Peccei-Quinn-like symmetry [42,43]. As a result, a mass for the $\phi_{0}$ 
meson is absent. The latter plays the role of an "electroweak axion". To see that, if we set $g_{2}=0$, the Higgs particle masses become:

$$
m_{\chi_{0}}=2 m_{b}, m_{\chi_{3}}=2 m_{t}, m_{\chi^{ \pm}}=2 m, m_{\phi_{0}}=0,
$$

where we used Equation (76) to get the relation

$$
\left.\frac{g_{2}}{\cos 2 \theta^{\prime}}\right|_{g_{2}=0}=2 g_{3}
$$

as well as the gap-Equations (74) and (75) to obtain that

$$
2 g_{3}=\left(m_{t}^{2}-m_{b}^{2}\right) \bar{g}^{2} \bar{C}_{2}
$$

at $g_{2}=0$. Now, relations of Equation (100) agree with the Nambu sum rule. In other words, the $U(1)_{A}$ breaking $\propto g_{2}$ accounts for the deviation from the canonical Nambu sum rule expressed by Equations (96) and (97).

We proceed to analyse the quark content of the composite Higgs particles as a possible SM-type Higgs particle with a mass that agrees with the empirical value. For example, let us take the neutral bound state $\bar{b} b$ described by the field function,

$$
\begin{aligned}
\chi_{0} & \propto \tilde{\sigma}_{0}=\sigma_{0} \cos \theta^{\prime}+\sigma_{3} \sin \theta^{\prime} \\
& \propto \bar{t} t\left[\left(g_{1}+g_{2}+g_{3}\right) \cos \theta^{\prime}+\left(g_{1}-g_{2}+g_{3}\right) \sin \theta^{\prime}\right] \\
& +\bar{b} b\left[\left(g_{1}+g_{2}-g_{3}\right) \cos \theta^{\prime}-\left(g_{1}-g_{2}-g_{3}\right) \sin \theta^{\prime}\right] \\
& \propto \bar{b} b
\end{aligned}
$$

where we used Equation (28) and that at $g_{2}=0$ and $m_{0} \neq m_{3}, \theta^{\prime}=-\pi / 4$, as seen from Equation (76). Likewise, the quark content of the remaining states can be made explicit. Hence, a light composite Higgs boson is built mainly of $\bar{b} b$ condensates with some proportion of $\bar{t} t$ due to the interaction $\propto g_{2}$. Accordingly an increase in its mass occurs in the interval $2 m_{b}<m_{\chi_{0}}<m_{t}$. Notice that if $m_{\chi_{0}} \ll \Lambda$, we can rely on the renormalization group to improve predictions obtained above for the low-energy Higgs masses. This is accomplished by re-summing leading logarithmic corrections to arbitrary loop order, for instance.

\section{Gauge Bosons}

The integration over the high-frequency components of the quark fields induces scale-dependent kinetic terms for the gauge fields that are not in the standard form. To recover the Yang-Mills part as in the Standard Model at $\mu<<\Lambda$, the gauge fields and their quark couplings must be rescaled, $g_{R}=Z_{A}^{1 / 2} g, g_{R}^{\prime}=Z_{B}^{1 / 2} g^{\prime}$; here, $g$ and $g^{\prime}$ stand for the renormalized couplings, fulfilling $g^{\prime} / g=\tan \theta_{W}, e=g \sin \theta_{W}, \sin ^{2} \theta_{W}=0.23$, and $Z_{A}, Z_{B}$ are $\mu$ - and $\Lambda$-dependent renormalizations, as will be discussed now.

Consider the effective Lagrangian whose kinetic part follows from Equations (6) and (62):

$$
\mathcal{L}_{\text {gauge }}^{\text {kin }}=\mathcal{L}_{\mathrm{YM}}+\frac{\bar{C}_{2}}{96} \operatorname{tr}\left(F_{\mu \nu}^{2}\right)+\frac{\bar{C}_{2}}{256} \operatorname{tr}\left(\left[\gamma^{\mu}, \gamma^{v}\right] F_{\mu \nu}\right)^{2},
$$

in which the trace over color degrees of freedom yields a factor $N_{c}$ that is absorbed in $\bar{C}_{2}$. Therefore, in the equation above, "tr" stands for the trace over SU(2) and Dirac gamma matrices. Moreover, the last term stems from the $Y^{2}$ part in Equation (62). This Lagrangian may be simplified as [36]

$$
\mathcal{L}_{\text {gauge }}^{\text {kin }}=\mathcal{L}_{\mathrm{YM}}-\frac{\bar{C}_{2}}{48} \operatorname{tr}\left(F_{\mu \nu}^{2}\right)
$$


and by integrating out the short-distance components of quark fields, one obtains a lowenergy correction to the Yang-Mills part of the Lagrangian density which, after evaluation of the trace, yields:

$$
\operatorname{tr}\left(F_{\mu v}^{2}\right)=2 \operatorname{tr}_{f}\left[g^{\prime 2}\left(Q^{2}+Y_{L}^{2}\right) B_{\mu \nu}^{2}+g^{2} G_{\mu v}^{2}\right]
$$

and considering that $G^{\mu v}=G_{i}^{\mu v} T_{i}$, and $Y_{L}=1 / 6$, this gives

$$
\mathcal{L}_{\text {gauge }}^{\text {kin }}=\mathcal{L}_{\text {YM }}-\frac{g^{2} \bar{C}_{2}}{48}\left(\frac{11}{9} \tan ^{2} \theta_{W} B_{\mu v}^{2}+\vec{G}_{\mu v}^{2}\right) .
$$

In terms of physical gauge fields, it may be rewritten as

$$
\begin{aligned}
\mathcal{L}_{\text {gauge }}^{\text {kin }}=-\frac{1}{4} & {\left[\left(\frac{1}{Z_{B}}+\frac{g^{2} \bar{C}_{2}}{12} \frac{11}{9} \tan ^{2} \theta_{W}\right) B_{\mu v}^{2}\right.} \\
+ & \left.\left(\frac{1}{Z_{A}}+\frac{g^{2} \bar{C}_{2}}{12}\right) \vec{G}_{\mu v}^{2}\right] .
\end{aligned}
$$

The free real constants $Z_{A}$ and $Z_{B}$ are fixed by requiring the standard form for kinetic terms of gauge-fields

$$
Z_{A}^{-1}=1-\frac{g^{2} \bar{C}_{2}}{12}, \quad Z_{B}^{-1}=1-\frac{g^{2} \bar{C}_{2}}{12} \frac{11}{9} \tan ^{2} \theta_{W} .
$$

Additionally, by a rescaling of the gauge fields, we have seen that the kinetic term does not change with $\mu$, unlike the masses of the gauge fields generated by the Higgs mechanism

$$
\mathcal{L}_{\text {gauge }}^{\text {mass }}=\frac{1}{4} g^{2} m^{2} \bar{C}_{2}\left(W_{\mu}^{+} W_{\mu}^{-}+\frac{Z_{\mu}^{2}}{2 \cos ^{2} \theta_{W}}\right),
$$

that depends on $\mu$ through $\bar{C}_{2}$. The gauge field masses are

$$
m_{W}=\frac{1}{2} g m \bar{C}_{2}^{1 / 2}=m_{Z} \cos \theta_{W}
$$

which may be compared with the SM result $m_{W}=g v / 2, v$ being the vacuum-expectation value of the Higgs field. Combining these results yields

$$
v=m \sqrt{\bar{C}_{2}}=254.6 \mathrm{GeV}
$$

Given that, we may calculate the ratio $\Lambda / \mu$ :

$$
\frac{\Lambda}{\mu}=\exp \left[\frac{(2 \pi v)^{2}}{N_{c}\left(m_{t}^{2}+m_{b}^{2}\right)}\right]=2.345 \times 10^{12} .
$$

At $\mu=\Lambda_{E W}$, we have $\Lambda \simeq 0.58 \times 10^{15} \mathrm{GeV}$, which suggests the possible role of GUT in the appearance of four-quark interactions through GUT symmetry-breaking.

\section{Numerical Results}

The model we considered here has five free parameters: $g_{1}, g_{2}, g_{3}, \Lambda, \mu$. They will be fixed at the SM scale, $\mu=\Lambda_{E W}=246 \mathrm{GeV}$. Thus, using Equation (113), we get $\Lambda=0.58 \times 10^{15} \mathrm{GeV}$. Moreover, we assume that $g_{1}, g_{2}, g_{3}$ are chosen so as to obtain the phenomenologically consistent solutions to gap equations. In other words, one chooses the free parameters to get the experimental values of quark masses, $m_{t}=173 \pm 0.4 \mathrm{GeV}$, $m_{b}=4.18_{-0.03}^{+0.04} \mathrm{GeV}$. We also require the Higgs mass state to be $m_{\chi_{0}}=125 \mathrm{GeV}$. We may 
verify that this can be accomplished under compatible conditions. The Higgs state spectrum as displayed in Equations (91)-(94) depends on three independent parameters, namely $m_{t}$ and $m_{b}$, and the ratio $g_{3} / g_{2}$, which we replace by the dimensionless parameter $a$

$$
\frac{g_{3}}{g_{2}}=a \tan 2 \theta
$$

The other parameters in Equations (91)-(94) are eliminated using Equation (85). Moreover, the angle $\theta$ and the mass $m$ in Equations (91)-(94) depend on the quark masses $m_{t}$ and $m_{b}$

$$
\tan 2 \theta=\frac{m_{t}^{2}-m_{b}^{2}}{2 m_{t} m_{b}}, \quad m^{2}=\frac{1}{2}\left(m_{t}^{2}+m_{b}^{2}\right),
$$

which yields $\theta=43.6^{\circ}$, while $\theta^{\prime}$ can be written in terms of the quark masses and the parameter $a$ only. In addition, from Equation (76)

$$
\tan 2 \theta^{\prime}=(3-2 a) \tan 2 \theta,
$$

we see that $\theta^{\prime}<0$ if $a>3 / 2$. The mass formulas (91)-(94) can then be written as

$$
\begin{aligned}
& m_{\chi_{0}}^{2}=\frac{2 m^{2}}{a-1}(2 a-1-\Delta), \\
& m_{\chi_{3}}^{2}=\frac{2 m^{2}}{a-1}(2 a-1+\Delta), \\
& m_{\phi_{0}}^{2}=\frac{4 m^{2}}{a-1}, \\
& m_{\chi^{ \pm}}^{2}=\frac{4 m^{2} a}{a-1},
\end{aligned}
$$

where

$$
\Delta=\sqrt{\cos ^{2} 2 \theta+(3-2 a)^{2} \sin ^{2} 2 \theta} .
$$

By fixing the parameter $a$ with mass of the standard Higgs state: $m_{\chi_{0}}=125 \mathrm{GeV} \rightarrow$ $\mathrm{a}=4.84$, we obtain the following estimates: $m_{\chi_{3}}=346 \mathrm{GeV}, m_{\chi^{ \pm}}=275 \mathrm{GeV}, m_{\phi_{0}}=125 \mathrm{GeV}$, and from Equation (116), $\theta^{\prime}=-44.8^{\circ}$. The values of four-Fermi couplings follow in a straightforward manner. Using Equation (114), we have $g_{3} / g_{2}=10^{2}$, so $g_{3} \gg g_{2}$. Therefore, using the gap-Equations (74) and (75), we may calculate $g_{1}$ and $g_{3}$. These equations show, however, that the coupling constants of the model must be extremely fine-tuned when $\mu^{2} \ll \Lambda^{2}$. Explicitly,

$$
\begin{aligned}
& g_{1}=g_{c}+\mathcal{O}\left(\frac{\mu^{2}}{\Lambda^{2}}\right), \quad g_{c}=\frac{4 \pi^{2}}{N_{c} \Lambda^{2}}=3.9 \times 10^{-29} \mathrm{GeV}^{-2}, \\
& g_{3}=g_{c} \frac{m_{t}^{2}-m_{b}^{2}}{2 \Lambda^{2}} \ln \frac{\Lambda^{2}}{\mu^{2}}+\mathcal{O}\left(\frac{\mu^{2}}{\Lambda^{2}}\right),
\end{aligned}
$$

which is the usual fine-tuning or the gauge-hierarchy problem of the SM, here isolated in the gap equation sector of the NJL approach. Our results imply that $g_{1}$ nearly coincides with the critical value $g_{c}$, and $g_{3}$ to the value $2.5 g_{c} \times 10^{-24} \simeq 1.0 \times 10^{-52} \mathrm{GeV}^{-2}$. The couplings must be fine-tuned to within

$$
\frac{g_{1}}{g_{c}}: \frac{g_{2}}{g_{c}}: \frac{g_{3}}{g_{c}} \sim 1: 10^{-26}: 10^{-24}
$$

of the critical value $g_{c}$. Finally, in spite of a good agreement in the estimates of both the quark masses and the ground Higgs state, the values for the neutral, $\phi_{0}$, mass and for the 
mass of the charged, $\chi^{ \pm}$Higgs states are likely experimentally disfavoured. More work needs to be done in refining the calculation of the mass spectrum using the renormalization group approach.

\section{Conclusions}

The purpose of this work has been to display in a pedagogical way the relevance of using the Schwinger-DeWitt background field method to address the Miransky, Tanabashi and Yamawaki (MTY) model Lagrangian of compositeness [17]. For that, we adopted a completely analytical path that preserved and helped to explore the symmetry content of the model at each step undertaken to express it in the form of a two-Higgs-doublet model. This path led ultimately to a CP-preserving spectrum of collective states, their interactions, and Yukawa couplings. By appropriately fixing the symmetry defining interaction parameters, we elucidated the structure of the collective state spectrum in detail at the electroweak scale, uncovering novel aspects. In the following, we summarize the relevant steps and conclusions reached.

We extended the vacuum structure of the MTY model proposed in [17] by performing a "minimal bosonization" in which no new coupling constants are generated. We used the Schwinger-DeWitt method to derive an effective Lagrangian whose induced parameters explicitly depend on two scales $\Lambda$ and $\mu$ and vanish as $\mu \rightarrow \Lambda$ (the so-called compositeness condition). We bypassed the computation of the fermion one-loop diagrams in the NambuGoldstone phase by starting from the massless fermion loop in the symmetric phase which lead to an effective Lagrangian with simpler gap equations. Then, we analyzed the main consequences of our model to the spectrum of composite Higgs states.

As repeatedly mentioned, an important result is that the empirical value for the Standard model Higgs mass can be accommodated in the spectrum, contrary to the customary wisdom that this model is unable to reproduce the current experimental value of the Higgs mass. The underlying quark content of the collective states could be scrutinized, see Equation (103). Within our approach, the standard Higgs is not purely a $\bar{t} t$-bound state, but rather, it has contributions from light bottom quarks. The results show that the $\theta^{\prime}$ mixing angle is about $-\pi / 4$, and thus, the $\bar{b} b$ contribution is dominant in the Higgs $\chi_{0}$ field.

In addition, we predicted the existence of a heavier neutral state which would be the Nambu partner of the standard Higgs within the Nambu sum rule, along with a neutral pseudoscalar state, $\phi_{0}$, that in the limit of a global $U(1)_{A}$ symmetry is a Goldstone mode. We have seen that there occurs a violation in the standard picture of Nambu partners due to the presence of a fifth, $\phi_{0}$, neutral boson in Equation (98). The resulting "electroweak axion" acquires mass through $U(1)_{A}$ symmetry-breaking interactions which are a subleading effect in a large $N_{c}$ counting. Because a coupling governed by small $g_{2}$ (as compared to $g_{1}$ ) is necessary for generating the mass of this state, we conclude that the standard expressions for the Nambu sum rule are only valid at leading order $1 / N_{c}$, and we have shown how the rule gets modified due to subleading corrections.

We obtained an accidental degeneracy between the main Higgs $\chi_{0}$ and $\phi_{0}, m_{\chi_{0}} \simeq m_{\phi_{0}}$. We expect that it may be lifted by considering quantum corrections, since in 2HDM models there appears only a genuine degeneracy, in the inert doublet model, in which an exact discrete $Z_{2}$ symmetry is present in the vacuum. In that case, all particles of the SM and one of the two-Higgs-doublet components are even, and the second Higgs doublet component is odd under such multiplicative discrete symmetry [40,41]. In these works, the double Higgs models are classified by the $Z_{2}, U(1)_{A}, S O(3)$, and three classes of generalized CP-symmetries. The inert model is not realized in the present case because it would require $\operatorname{Re} \lambda_{5}=\lambda_{1}-\lambda_{3}-\lambda_{4}$ and $m_{12}^{2}=0$ (or equivalently, $g_{3}=0$ ) and $g_{2}=0$ are simultaneously fulfilled.

The main drawback of our approach is that the dynamics lies at a very high-energy scale $\Lambda \sim 10^{15} \mathrm{GeV}$ which corresponds to the GUT scale $10^{15} \mathrm{GeV}$. Despite rendering credit to the scenarios generated by GUT physics, the model is very fine-tuned. Such a feature is isolated in the gap equations: if one tunes the couplings $g_{1}, g_{2}, g_{3}$ to the solution, the quadratic divergences are cancelled in other amplitudes. 
As a future work, we mention considering electroweak and strong corrections for the spectrum of states, which may be achieved using renormalization group techniques.

Author Contributions: Writing—review and editing, A.A.O., B.H., A.H.B. and M.S. All authors have read and agreed to the published version of the manuscript.

Funding: This article is based upon work from COST Action 16201, supported by COST (European Cooperation in Science and Technology, www.cost.eu (accessed on 19 January 2019)). We acknowledge support from CFisUC and Fundação para a Ciência e Tecnologia through the project UID/FIS/04564/ 2020, and grant CERN/FIS-COM/0035/2019. M. Sampaio acknowledges a research grant from CNPq.

Institutional Review Board Statement: Not applicable.

Acknowledgments: A. A. Osipov would like to thank C. T. Hill for his interest in this study and valuable correspondence.

Conflicts of Interest: The authors declare no conflict of interest.

\section{Appendix A. Diagonalization of the Higgs States}

The diagonalization of a quadratic form

$$
\Omega_{y}^{x}(a, b, c / 2)=(x, y)\left(\begin{array}{cc}
a & c / 2 \\
c / 2 & b
\end{array}\right)\left(\begin{array}{l}
x \\
y
\end{array}\right)
$$

is effected by an orthogonal transformation $R(\theta)$ to the new variables $(\tilde{x}, \tilde{y})$

$$
\left(\begin{array}{l}
x \\
y
\end{array}\right)=\left(\begin{array}{cc}
\cos \theta & \sin \theta \\
-\sin \theta & \cos \theta
\end{array}\right)\left(\begin{array}{l}
\tilde{x} \\
\tilde{y}
\end{array}\right)=R(\theta)\left(\begin{array}{l}
\tilde{x} \\
\tilde{y}
\end{array}\right) .
$$

The off-diagonal terms are removed using the condition

$$
\tan 2 \theta=\frac{c}{b-a}
$$

and the diagonal pieces are

$$
\begin{aligned}
& \Omega_{y}^{x}(a, b, c / 2)=\Omega_{\tilde{y}}^{\tilde{x}}\left(a_{11}, a_{22}, 0\right), \\
& a_{11}=\frac{1}{2}\left[a+b+(a-b) \sqrt{1+\tan ^{2} 2 \theta}\right], \\
& a_{22}=\frac{1}{2}\left[a+b-(a-b) \sqrt{1+\tan ^{2} 2 \theta}\right] .
\end{aligned}
$$

This is used to obtain the diagonal form of the quadratic terms of the potential Equation (64), after applying the redefinitions $\sigma_{0} \rightarrow \sigma_{0}-m_{0}$ and $\sigma_{3} \rightarrow \sigma_{3}-m_{3}$,

$$
V_{H}^{(2)}=\Omega_{\pi_{3}}^{\pi_{0}}+\Omega_{\sigma_{0}}^{\sigma_{3}}+\Omega_{\sigma_{1}}^{\pi_{2}}+\Omega_{\pi_{1}}^{\sigma_{2}},
$$

with the arguments of the quadratic forms denoted accordingly

$$
\begin{aligned}
& a_{11}^{\pi_{0}, \pi_{3}}=\frac{1}{\bar{g}^{2}}\left(g_{1}+g_{2}\right)+m^{2} \bar{C}_{2}-\bar{C}_{1}, \\
& a_{22}^{\pi_{0}, \pi_{3}}=\frac{1}{\bar{g}^{2}}\left(g_{1}-g_{2}\right)+m^{2} \bar{C}_{2}-\bar{C}_{1}, \\
& a_{12}^{\pi_{0}, \pi_{3}}=2 m_{0} m_{3} \bar{C}_{2}-\frac{g_{3}}{\bar{g}^{2}} .
\end{aligned}
$$




$$
\begin{gathered}
a_{11}^{\sigma_{3}, \sigma_{0}}=\frac{1}{\bar{g}^{2}}\left(g_{1}+g_{2}\right)+3 m^{2} \bar{C}_{2}-\bar{C}_{1}, \\
a_{22}^{\sigma_{3}, \sigma_{0}}=\frac{1}{\bar{g}^{2}}\left(g_{1}-g_{2}\right)+3 m^{2} \bar{C}_{2}-\bar{C}_{1}, \\
a_{12}^{\sigma_{3}, \sigma_{0}}=6 m_{0} m_{3} \bar{C}_{2}-\frac{g_{3}}{\bar{g}^{2}} . \\
a_{11}^{\pi_{2}, \sigma_{1}}=\frac{1}{\bar{g}^{2}}\left(g_{1}-g_{2}\right)+\left(m_{0}^{2}+3 m_{3}^{2}\right) \bar{C}_{2}-\bar{C}_{1}, \\
a_{22}^{\pi_{2}, \sigma_{1}}=\frac{1}{\bar{g}^{2}}\left(g_{1}+g_{2}\right)+\left(3 m_{0}^{2}+m_{3}^{2}\right) \bar{C}_{2}-\bar{C}_{1}, \\
a_{12}^{\pi_{2}, \sigma_{1}}=\frac{g_{3}}{\bar{g}^{2}} . \\
a_{11}^{\sigma_{2}, \pi_{1}}=\frac{1}{\bar{g}^{2}}\left(g_{1}+g_{2}\right)+\left(3 m_{0}^{2}+m_{3}^{2}\right) \bar{C}_{2}-\bar{C}_{1}, \\
a_{22}^{\sigma_{2}, \pi_{1}}=\frac{1}{\bar{g}^{2}}\left(g_{1}-g_{2}\right)+\left(m_{0}^{2}+3 m_{3}^{2}\right) \bar{C}_{2}-\bar{C}_{1}, \\
a_{12}^{\sigma_{2}, \pi_{1}}=-\frac{g_{3}}{\bar{g}^{2}},
\end{gathered}
$$

recalling the definition $m^{2}=m_{0}^{2}+m_{3}^{2}$. The diagonalization leads to

$$
\theta_{\pi_{0} \pi_{3}}=\theta_{\pi_{2} \sigma_{1}}=\theta_{\sigma_{2} \pi_{1}} \equiv \theta,
$$

where

$$
\tan 2 \theta=\frac{m_{t}^{2}-m_{b}^{2}}{2 m_{t} m_{b}}, \quad \text { or } \quad \tan \theta=\frac{m_{3}}{m_{0}} .
$$

To obtain these expressions use of the gap Equations (72) and (73) has been made. A different angle is obtained for $\Omega_{\sigma_{0}}^{\sigma_{3}}$

$$
\theta_{\sigma_{3} \sigma_{0}} \equiv \theta^{\prime}
$$

with relation

$$
\tan 2 \theta^{\prime}=3 \frac{m_{t}^{2}-m_{b}^{2}}{2 m_{t} m_{b}}-2 \frac{g_{3}}{g_{2}}=3 \tan 2 \theta-2 \frac{g_{3}}{g_{2}} .
$$

The diagonalized quadratic piece of the potential $V_{H}$ is

$$
\begin{aligned}
V_{H}^{(2)} & =\frac{g_{2}}{\bar{g}^{2}} \frac{m_{t}^{2}+m_{b}^{2}}{m_{t} m_{b}} \tilde{\pi}_{0}^{2}+\frac{2 g_{3}}{\bar{g}^{2}} \frac{m_{t}^{2}+m_{b}^{2}}{m_{t}^{2}-m_{b}^{2}}\left(\tilde{\sigma}_{1}^{2}+\tilde{\sigma}_{2}^{2}\right) \\
& +\left[\left(m_{t}^{2}+m_{b}^{2}\right) \bar{C}_{2}+\frac{g_{2}}{\bar{g}^{2}}\left(\frac{1}{\cos 2 \theta}-\frac{1}{\cos 2 \theta^{\prime}}\right)\right] \tilde{\sigma}_{0}^{2} \\
& +\left[\left(m_{t}^{2}+m_{b}^{2}\right) \bar{C}_{2}+\frac{g_{2}}{\bar{g}^{2}}\left(\frac{1}{\cos 2 \theta}+\frac{1}{\cos 2 \theta^{\prime}}\right)\right] \tilde{\sigma}_{3}^{2} .
\end{aligned}
$$

The coefficients (masses) associated to the bosonic variables given in $V_{H}^{(2)}$ are related to the initial doublets Equation (30) by the orthogonal transformation

$$
\left(\begin{array}{l}
\Phi_{1} \\
\Phi_{2}
\end{array}\right)=\left(\begin{array}{cc}
\cos \theta & \sin \theta \\
-\sin \theta & \cos \theta
\end{array}\right)\left(\begin{array}{c}
\tilde{\Phi}_{1} \\
\tilde{\Phi}_{2}
\end{array}\right)
$$


where the transformed states are

$$
\tilde{\Phi}_{1}=\left(\begin{array}{c}
\tilde{\pi}_{2}+i \tilde{\pi}_{1} \\
\sigma_{0}^{\prime}-i \tilde{\pi}_{3}
\end{array}\right), \quad \tilde{\Phi}_{2}=\left(\begin{array}{c}
\tilde{\sigma}_{1}-i \tilde{\sigma}_{2} \\
-\sigma_{3}^{\prime}+i \tilde{\pi}_{0}
\end{array}\right) .
$$

Here $\sigma_{0}^{\prime}$ and $\sigma_{3}^{\prime}$ are related to the physical neutral scalar states $\tilde{\sigma}_{0}, \tilde{\sigma}_{3}$ through

$$
\left(\begin{array}{l}
\sigma_{3}^{\prime} \\
\sigma_{0}^{\prime}
\end{array}\right) \equiv\left(\begin{array}{cc}
\cos \alpha & -\sin \alpha \\
\sin \alpha & \cos \alpha
\end{array}\right)\left(\begin{array}{l}
\tilde{\sigma}_{3} \\
\tilde{\sigma}_{0}
\end{array}\right) .
$$

with $\alpha=\theta-\theta^{\prime}$.

These expressions diagonalize the quadratic part of the potential $V_{H}$, under the assumption that the vacuum state is correctly determined. However when one minimizes the potential to obtain the ground state, one must take into consideration that the fields may acquire non-zero vacuum expectation values, and thus one must redefine the variables in Equation (A15) $\sigma_{0} \rightarrow \sigma_{0}-m_{0}$, and $\sigma_{3} \rightarrow \sigma_{3}-m_{3}$ as in Equation (79). These substitutions do not alter $\tilde{\Phi}_{2}=H_{2}$, since

$$
\begin{aligned}
\left(\begin{array}{c}
\sigma_{3}^{\prime} \\
\sigma_{0}^{\prime}
\end{array}\right) & \rightarrow\left(\begin{array}{c}
\sigma_{3}^{\prime} \\
\sigma_{0}^{\prime}
\end{array}\right)-R(-\alpha) R\left(-\theta^{\prime}\right)\left(\begin{array}{l}
m_{3} \\
m_{0}
\end{array}\right) \\
& =\left(\begin{array}{c}
\sigma_{3}^{\prime} \\
\sigma_{0}^{\prime}
\end{array}\right)-R(-\theta)\left(\begin{array}{l}
m_{3} \\
m_{0}
\end{array}\right)=\left(\begin{array}{c}
\sigma_{3}^{\prime} \\
\sigma_{0}^{\prime}
\end{array}\right)-\left(\begin{array}{c}
0 \\
m
\end{array}\right),
\end{aligned}
$$

where the relations

$$
\cos \theta=\frac{m_{0}}{m}, \quad \sin \theta=\frac{m_{3}}{m} .
$$

were used in the last stage. However $\tilde{\Phi}_{1}$ changes to $H_{1}$ with a (real and negative) vacuum expectation value $-m$ in the lower component. The vacuum expectation value of $H_{2}$ is zero.

\section{References}

1. Micco, B.D.; Gouzevitch, M.; Mazzitelli, J.; Vernieri, C. Higgs boson potential at colliders: Status and perspectives. Rev. Phys. 2020, 5, 100045. [CrossRef]

2. Heinrich, G. Collider Physics at the Precision Frontier. Phys. Rep. 2021, 922, 1-69. [CrossRef]

3. Zyla, P.A.; Barnett, R.M.; Beringer, J.; Dahl, O.; Dwyer, D.A.; Groom, D.E.; Lin, C.-J.; Lugovsky, K.S.; Pianori, E.; Robinson, D.J.; et al. Review of Particle Physics. Prog. Theor. Exp. Phys. 2020, 2020, 083C01. [CrossRef]

4. Liu, D.; Low, I.; Yin, Z. Universal Imprints of a Pseudo-Nambu-Goldstone Higgs Boson. Phys. Rev. Lett. 2018, 121, 261802. [CrossRef] [PubMed]

5. Witzel, O. Review on Composite Higgs Models. PoS LATTICE 2019. [CrossRef]

6. Kaplan, D.B.; Georgi, H.; Dimopoulos, S. Composite Higgs scalars. Phys. Lett. B 1984, 136, 187-190. [CrossRef]

7. Georgi, H.; Kaplan, D.B.; Galison, P. Calculation of the composite Higgs mass. Phys. Lett. B 1984, 143, 152-154. [CrossRef]

8. Georgi, H.; Kaplan, D.B. Composite Higgs and custodial SU(2). Phys. Lett. B 1984, 145, 216-220. [CrossRef]

9. Andersen, J.R.; Antipin, O.; Azuelos, G.; Debbio, L.D.; Nobile, E.D.; Di Chiara, S.; Hapola, T.; Järvinen, M.; Lowdon, P.J.; Maravin, Y.; et al. Discovering Technicolor. Eur. Phys. J. Plus 2011, 126, 81. [CrossRef]

10. Keus, V.; Koivunen, N.; Tuominen, K. Singlet scalar and 2HDM extensions of the Standard Model: CP-violation and constraints from $(g-2)_{\mu}$ and eEDM. J. High Energy Phys. 2018, 9, 59. [CrossRef]

11. Ko, P.; Omura, Y.; Yu, C. Two Higgs doublet models with local $U(1)_{H}$ [CrossRef] gauge symmetry and dark matter. J. High Energy Phys. 2014, 11, 54 .

12. Terazawa, H.; Chikashige, Y.; Akama, K. Unified Model of the Nambu-Jona-Lasinio Type for All Elementary Particle Forces. Phys. Rev. D 1977, 15, 480-487. [CrossRef]

13. Terazawa, H. Subquark Model of Leptons and Quarks. Phys. Rev. D 1980, 22, 184-199. [CrossRef]

14. Terazawa, H. t-quark mass predicted from a sum rule for lepton and quark masses. Phys. Rev. D 1980, 22, 2921; Erratum in 1990, 41, 3541. [CrossRef]

15. Nambu, Y. Quasisupersymmetry, bootstrap symmetry breaking and fermion masses. In Proceedings of the 1988 International Workshop New Trends in Strong Coupling Gauge Theories, Nagoya, Japan, 24-27 August 1988; Bando, M., Muta, T., Yamawaki, K., Eds.; EFI Report No. 89-08, 1989 Unpublished; World Scientific: Singapore, 1989. [CrossRef]

16. Nambu, Y. New Theories in Physics: Proceedings of the XI Warsaw Symposium on Elementary Particle Physics, 23-27 May, 1988, Kazimierz, Poland; Ajduk, Z., Pokorski, S., Trautman, A., Eds.; World Scientific: Singapore, 1989; pp. 1-10. 
17. Miransky, V.A.; Tanabashi, M.; Yamawaki, K. Dynamical electroweak symmetry breaking with large anomalous dimension and $\mathrm{t}$ quark condensate, Phys. Lett. B 1989, 221, 177-183.

18. Miransky, V.A.; Tanabashi, M.; Yamawaki, K. Is the $\mathrm{t}$ quark responsible for the mass of $\mathrm{W}$ and Z bosons? Mod. Phys. Lett. A 1989, 4, 1043-1053. [CrossRef]

19. Bardeen, W.A.; Hill, C.T.; Lindner, M. Minimal dynamical symmetry breaking of the standard model. Phys. Rev. D 1990, 41, 1647-1660. [CrossRef]

20. Luty, M.A. Dynamical electroweak symmetry breaking with two composite Higgs doublets. Phys. Rev. D 1990, 41, 2893. [CrossRef]

21. Suzuki, M. Composite Higgs bosons in the Nambu-Jona-Lasinio model. Phys. Rev. D 1990, 41, 3457-3463. [CrossRef]

22. Harada, M.; Kitazawa, N. Vacuum alignment in the top quark condensation. Phys. Lett. B 1991, 257, 383-387. [CrossRef] [PubMed]

23. Cvetic, G. Top-quark condensation. Rev. Mod. Phys. 1999, 71, 513-574. [CrossRef]

24. Hill, C.T.; Simmons, E.H. Strong Dynamics and Electroweak Symmetry Breaking. Phys. Rept. 2003, 381, 235-402; Erratum in 2004, 390, 553-554. [CrossRef]

25. Yamawaki, K. Origin of Mass-Horizons Expanding from the Nambu's Theory. arXiv 2016, arXiv:1605.01951. [CrossRef]

26. Fukano, H.S.; Tuominen, K. A hybrid 4th generation: Technicolor with top-seesaw. Phys. Rev. D 2012, 85, 095025.

27. Fukano, H.S.; Kurachi, M.; Matsuzaki, S.; Yamawaki, K. Higgs boson as a top-mode pseudo-Nambu-Goldstone boson. Phys. Rev. D 2014, 90, 055009. [CrossRef]

28. Fukano, S.; Kurachi, M.; Matsuzaki, S. Vacuum Alignment of the Top-Mode Pseudo-Nambu-Goldstone Boson Higgs Model. Phys. Rev. D 2015, 91, 115005. [CrossRef]

29. Nambu, Y.; Jona-Lasinio, G. Dynamical Model of Elementary Particles Based on an Analogy with Superconductivity. I. Phys. Rev. 1961, 122, 345-358. [CrossRef]

30. Nambu, Y.; Jona-Lasinio, G. Dynamical Model of Elementary Particles Based on an Analogy with Superconductivity. II. Phys. Rev. 1961, 124, 246-254. [CrossRef]

31. Nambu, Y. Fermion-Boson relations in BCS-type theories. Physica D 1985, 15, 147-151. [CrossRef]

32. Volovik, G.E.; Zubkov, M.A. Nambu sum rule and the relation between the masses of composite Higgs bosons. Phys. Rev. D 2013, 87, 075016. [CrossRef]

33. Volovik, G.E.; Zubkov, M.A. Nambu sum rule in the NJL models: From superfluidity to top quark condensation. Pisma $v$ ZhETF 2013, 97, 344. [CrossRef]

34. Geller, M.; Bar-Shalom, S.; Soni, A. Higgs-radion unification: Radius stabilization by an SU(2) bulk doublet and the $126 \mathrm{GeV}$ scalar. Phys. Rev. D 2014, 89, 035012. [CrossRef]

35. Yamawaki, K. Old wine in a new bottle: Technidilaton as the $125 \mathrm{GeV}$ Higgs. arXiv 2015, arXiv:1511.06883. [CrossRef]

36. Osipov, A.A.; Hiller, B.; Blin, A.H.; Palanca, F.; Moreira, J.; Sampaio, M. Top condensation model: A step towards the correct predictionof the Higgs mass. Eur. Phys. J. C 2020, 80, 1135.

37. 't Hooft, G. Computation of the quantum effects due to a four-dimensional pseudoparticle. Phys. Rev. D 1976, 14, 3432-3450; Erratum in 1978, 18, 2199. [CrossRef]

38. Eguchi, T. Nonrenormalizable Interactions and Eigenvalue Condition. Phys. Rev. D 1978, 17, 611-615. [CrossRef]

39. Branco, G.C.; Ferreira, P.M.; Lavoura, L.; Rebelo, M.N.; Sher, M.; Silva, J.P. Theory and phenomenology of two-Higgs-doublet models. Phys. Rep. 2012, 516, 1-102. [CrossRef]

40. Ferreira, P.M.; Haber, H.E.; Silva, J.P. Generalized CP symmetries and special regions of parameter space in the two-Higgs-doublet model. Phys. Rev. D 2009, 79, 116004. [CrossRef]

41. Haber, H.E.; Ogreid, O.M.; Osland, P.; Rebelo, M.N. Symmetries and Mass Degeneracies in the Scalar Sector. J. High Energy Phys. 2019, 1, 42. [CrossRef]

42. Peccei, R.D.; Quinn, H.R. CP Conservation in the Presence of Pseudoparticles. Phys. Rev. Lett. 1977, 38, 1440. [CrossRef]

43. Peccei, R.D.; Quinn, H.R. Constraints imposed by CP conservation in the presence of pseudoparticles. Phys. Rev. D 1977, 16, 1791-1797. [CrossRef]

44. Wilson, K.G.; Kogut, J. The renormalization group and the $\epsilon$ expansion. Phys. Rep. C 1974, 12, 75-199. [CrossRef]

45. Schwinger, J. On Gauge Invariance and Vacuum Polarization. Phys. Rev. 1951, 82, 664-679. [CrossRef]

46. DeWitt, B.S. Dynamical Theory of Groups and Fields; Gordon and Breach: New York, NY, USA, 1965. [CrossRef]

47. DeWitt, B.S. Quantum field theory in curved spacetime. Phys. Rep. 1975, 19, 295.

48. Ball, R.D. Chiral gauge theory. Phys. Rep. 1989, 182, 1-186. [CrossRef]

49. Osipov, A.A.; Volkov, M.K. Chiral transformations of spin-1 mesons in the non-symmetric vacuum. Ann. Phys. 2017, 382, 50-63. [CrossRef]

50. Osipov, A.A.; Khalifa, M.M. Catalysis of the $<\bar{b} b>$ Condensate in the Composite Higgs Model. JETP Lett. 2019, 110, 387. [CrossRef]

51. Osipov, A.A.; Khalifa, M.M. The Nambu Sum Rule in the Composite Two Higgs Doublet Model. Phys. Part. Nucl. Lett. 2020, 17, 296-302. 